EUROPEAN ORGANISATION FOR NUCLEAR RESEARCH

CERN-PPE/95-12

25 January 1995

\title{
A Study of B Meson Oscillations Using Dilepton Events
}

\author{
The OPAL Collaboration
}

\begin{abstract}
The time dependence of $B$ meson oscillations is studied using hadronic $Z^{0}$ decays with identified leptons in both thrust hemispheres. Decay times are reconstructed for each of the semileptonic $B$ decays by forming vertices which include the lepton and by estimating the $B$ meson momentum. The mass difference of the two mass eigenstates in the $B_{d}^{0}$ system, $\Delta m_{\mathrm{d}}$, is measured to be $0.462_{-0.053}^{+0.040}{ }_{-0.035}^{+0.052} \mathrm{ps}^{-1}$, where the first error is statistical and the second systematic. For the $\mathrm{B}_{\mathrm{s}}^{\mathrm{o}}$ system, a lower limit of $\Delta m_{\mathrm{s}}>2.2 \mathrm{ps}^{-1}$ at $95 \%$ C.L. is derived.
\end{abstract}

(To be submitted to Zeitschrift für Physik C) 


\section{The OPAL Collaboration}

R. Akers ${ }^{16}$, G. Alexander ${ }^{23}$, J. Allison ${ }^{16}$, K. Ametewee ${ }^{25}$, K.J. Anderson ${ }^{9}$, S. Arcelli ${ }^{2}$, S. Asai ${ }^{24}$, D. Axen ${ }^{29}$, G. Azuelos ${ }^{18, a}$, A.H. Ball ${ }^{17}$, E. Barberio ${ }^{26}$, R.J. Barlow ${ }^{16}$, R. Bartoldus ${ }^{3}$, J.R. Batley ${ }^{5}$, G. Beaudoin ${ }^{18}$, A. Beck ${ }^{23}$, G.A. Beck ${ }^{13}$, C. Beeston ${ }^{16}$, T. Behnke ${ }^{27}$, K.W. Bell ${ }^{20}$, G. Bella ${ }^{23}$, S. Bentvelsen ${ }^{8}$, P. Berlich ${ }^{10}$, S. Bethke ${ }^{32}$, O. Biebel ${ }^{32}$, I.J. Bloodworth ${ }^{1}$, P. Bock ${ }^{11}$, H.M. Bosch ${ }^{11}$, M. Boutemeur ${ }^{18}$, S. Braibant ${ }^{12}$, P. Bright-Thomas ${ }^{25}$, R.M. Brown ${ }^{20}$, A. Buijs ${ }^{8}$, H.J. Burckhart ${ }^{8}$, R. Bürgin ${ }^{10}$, C. Burgard ${ }^{27}$, N. Capdevielle ${ }^{18}$, P. Capiluppi ${ }^{2}$, R.K. Carnegie ${ }^{6}$, A.A. Carter $^{13}$, J.R. Carter ${ }^{5}$, C.Y. Chang ${ }^{17}$, C. Charlesworth ${ }^{6}$, D.G. Charlton ${ }^{1, b}$, S.L. Chu ${ }^{4}$,

P.E.L. Clarke ${ }^{15}$, J.C. Clayton ${ }^{1}$, S.G. Clowes $^{16}$, I. Cohen ${ }^{23}$, J.E. Conboy ${ }^{15}$, O.C. Cooke ${ }^{16}$, M. Cuffiani ${ }^{2}$, S. Dado ${ }^{22}$, C. Dallapiccola ${ }^{17}$, G.M. Dallavalle ${ }^{2}$, C. Darling ${ }^{31}$, S. De Jong ${ }^{12}$, L.A. del

Pozo $^{8}$, H. Deng ${ }^{17}$, M.Dittmar ${ }^{4}$, M.S. Dixit ${ }^{7}$, E. do Couto e Silva ${ }^{12}$, J.E. Duboscq ${ }^{8}$,

E. Duchovni ${ }^{26}$, G. Duckeck $^{8}$, I.P. Duerdoth ${ }^{16}$, U.C. Dunwoody ${ }^{5}$, J.E.G. Edwards ${ }^{16}$,

P.A.Elcombe ${ }^{5}$, P.G. Estabrooks ${ }^{6}$, E. Etzion ${ }^{23}$, H.G. Evans ${ }^{9}$, F. Fabbri ${ }^{2}$, B. Fabbro ${ }^{21}$, M. Fanti ${ }^{2}$, P. Fath ${ }^{11}$, M. Fierro ${ }^{2}$, M. Fincke-Keeler ${ }^{28}$, H.M. Fischer ${ }^{3}$, P. Fischer ${ }^{3}$, R. Folman ${ }^{26}$, D.G. Fong ${ }^{17}$,

M. Foucher ${ }^{17}$, H. Fukui ${ }^{24}$, A. Fürtjes ${ }^{8}$, P. Gagnon ${ }^{6}$, A. Gaidot ${ }^{21}$, J.W. Gary ${ }^{4}$, J. Gascon ${ }^{18}$, N.I. Geddes ${ }^{20}$, C. Geich-Gimbel ${ }^{3}$, S.W. Gensler ${ }^{9}$, F.X. Gentit ${ }^{21}$, T. Geralis ${ }^{20}$, G. Giacomelli ${ }^{2}$,

P. Giacomelli ${ }^{4}$, R. Giacomelli ${ }^{2}$, V. Gibson ${ }^{5}$, W.R. Gibson ${ }^{13}$, J.D. Gillies ${ }^{20}$, J. Goldberg ${ }^{22}$,

D.M. Gingrich ${ }^{30, a}$, M.J. Goodrick ${ }^{5}$, W. Gorn ${ }^{4}$, C. Grandi' ${ }^{2}$, E. Gross ${ }^{26}$, J. Hagemann ${ }^{27}$, G.G. Hanson ${ }^{12}$, M.Hansroul ${ }^{8}$, C.K. Hargrove ${ }^{7}$, P.A. Hart ${ }^{9}$, M. Hauschild ${ }^{8}$, C.M. Hawkes ${ }^{8}$, E. Heflin ${ }^{4}$, R.J.Hemingway ${ }^{6}$, G.Herten ${ }^{10}$, R.D. Heuer ${ }^{8}$, J.C.Hill ${ }^{5}$, S.J. Hillier ${ }^{8}$, T. Hilse ${ }^{10}$, P.R.Hobson ${ }^{25}$, D. Hochman ${ }^{26}$, R.J. Homer ${ }^{1}$, A.K. Honma ${ }^{28, a}$, R. Howard ${ }^{29}$,

R.E. Hughes-Jones ${ }^{16}$, P. Igo-Kemenes ${ }^{11}$, D.C. Imrie ${ }^{25}$, A. Jawahery ${ }^{17}$, P.W. Jeffreys ${ }^{20}$, H. Jeremie ${ }^{18}$, M. Jimack ${ }^{1}$, M. Jones ${ }^{6}$, R.W.L. Jones ${ }^{8}$, P. Jovanovic ${ }^{1}$, C. Jui ${ }^{4}$, D. Karlen ${ }^{6}$, J. Kanzaki ${ }^{24}$, K. Kawagoe ${ }^{24}$, T. Kawamoto ${ }^{24}$, R.K. Keeler ${ }^{28}$, R.G. Kellogg ${ }^{17}$, B.W. Kennedy ${ }^{20}$, B. King ${ }^{8}$, J. King ${ }^{13}$, J. Kirk ${ }^{29}$, S. Kluth ${ }^{5}$, T. Kobayashi ${ }^{24}$, M. Kobel ${ }^{10}$, D.S. Koetke ${ }^{6}$, T.P. Kokott ${ }^{3}$, S. Komamiya ${ }^{24}$, R. Kowalewski ${ }^{8}$, T. Kress ${ }^{11}$, P. Krieger ${ }^{6}$, J. von Krogh ${ }^{11}$, P. Kyberd ${ }^{13}$, G.D. Lafferty ${ }^{16}$, H. Lafoux ${ }^{8}$, R. Lahmann ${ }^{17}$, W.P. Lai ${ }^{19}$, J. Lauber ${ }^{8}$, J.G. Layter ${ }^{4}$, P. Leblanc ${ }^{18}$, A.M. Lee ${ }^{31}$, E. Lefebvre ${ }^{18}$, D. Lellouch ${ }^{26}$, C. Leroy ${ }^{18}$, J. Letts ${ }^{2}$, L. Levinson ${ }^{26}$, S.L. Lloyd ${ }^{13}$, F.K. Loebinger ${ }^{16}$, G.D. Long ${ }^{17}$, B. Lorazo ${ }^{18}$, M.J. Losty ${ }^{7}$, X.C. Lou ${ }^{8}$, J. Ludwig ${ }^{10}$, A. Luig ${ }^{10}$, M. Mannelli ${ }^{8}$, S. Marcellini ${ }^{2}$, C. Markus ${ }^{3}$, A.J. Martin ${ }^{13}$, J.P. Martin ${ }^{18}$, T. Mashimo ${ }^{24}$, W. Matthews ${ }^{25}$, P. Mättig ${ }^{3}$, U. Maur ${ }^{3}$, J. McKenna ${ }^{29}$, T.J. McMahon ${ }^{1}$, A.I. $\mathrm{McNab}^{13}$, F. Meijers ${ }^{8}$, F.S. Merritt ${ }^{9}$, H. $\mathrm{Mes}^{7}$, A. Michelini ${ }^{8}$, R.P. Middleton ${ }^{20}$, G. Mikenberg ${ }^{26}$, D.J. Miller ${ }^{15}$, R. Mir $^{26}$, W. Mohr ${ }^{10}$, A. Montanari ${ }^{2}$, T. Mori ${ }^{24}$, M. Morii ${ }^{24}$, U. Müller ${ }^{3}$, B. Nellen ${ }^{3}$, B. Nijjhar ${ }^{16}$, S.W. O'Neale ${ }^{1}$, F.G. Oakham ${ }^{7}$, F. Odorici ${ }^{2}$, H.O. Ogren ${ }^{12}$, N.J. Oldershaw ${ }^{16}$, C.J. Oram ${ }^{28, a}$, M.J. Oreglia ${ }^{9}$, S. Orito ${ }^{24}$, F. Palmonari ${ }^{2}$, J.P. Pansart ${ }^{21}$,

G.N.Patrick ${ }^{20}$, M.J. Pearce ${ }^{1}$, P.D. Phillips ${ }^{16}$, J.E. Pilcher ${ }^{9}$, J.Pinfold ${ }^{30}$, D.E. Plane ${ }^{8}$, P. Poffenberger ${ }^{28}$, B. Poli ${ }^{2}$, A. Posthaus ${ }^{3}$, T.W. Pritchard ${ }^{13}$, H. Przysiezniak ${ }^{30}$,

M.W. Redmond ${ }^{8}$, D.L. Rees ${ }^{8}$, D. Rigby ${ }^{1}$, M.G. Rison ${ }^{5}$, S.A. Robins ${ }^{13}$, D. Robinson ${ }^{5}$, N. Rodning ${ }^{30}$, J.M. Roney ${ }^{28}$, E. Ros ${ }^{8}$, A.M.Rossi ${ }^{2}$, M. Rosvick ${ }^{28}$, P. Routenburg ${ }^{30}$, Y. Rozen ${ }^{8}$, K. Runge ${ }^{10}$, O. Runolfsson ${ }^{8}$, D.R. Rust ${ }^{12}$, M.Sasaki ${ }^{24}$, C.Sbarra ${ }^{2}$, A.D.Schaile ${ }^{8}$, O.Schaile ${ }^{10}$, F.Scharf ${ }^{3}$, P.Scharff-Hansen ${ }^{8}$, P.Schenk ${ }^{4}$, B. Schmitt ${ }^{3}$, M. Schröder ${ }^{8}$, H.C. Schultz-Coulon ${ }^{10}$, P.Schütz ${ }^{3}$, M.Schulz ${ }^{8}$, C.Schwick ${ }^{27}$, J.Schwiening ${ }^{3}$, W.G.Scott ${ }^{20}$, M.Settles ${ }^{12}$, T.G.Shears ${ }^{5}$, B.C.Shen ${ }^{4}$, C.H. Shepherd-Themistocleous ${ }^{7}$, P. Sherwood ${ }^{15}$, G.P. Siroli ${ }^{2}$, A. Skillman ${ }^{15}$, A.Skuja ${ }^{17}$, A.M.Smith ${ }^{8}$, T.J.Smith ${ }^{28}$, G.A.Snow ${ }^{17}$, R. Sobie ${ }^{28}$, S. Söldner-Rembold ${ }^{10}$, R.W.Springer ${ }^{30}$, M.Sproston ${ }^{20}$, A.Stahl ${ }^{3}$, M.Starks ${ }^{12}$, C.Stegmann ${ }^{10}$, K.Stephens ${ }^{16}$, J. Steuerer $^{28}$, B. Stockhausen ${ }^{3}$, D. Strom ${ }^{19}$, P.Szymanski ${ }^{20}$, R. Tafirout ${ }^{18}$, H. Takeda ${ }^{24}$, 


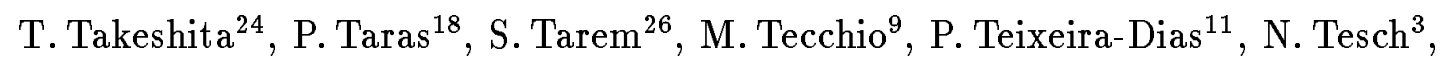
M.A. Thomson ${ }^{8}$, O. Tousignant ${ }^{18}$, S. Towers ${ }^{6}$, M. Tscheulin ${ }^{10}$, T. Tsukamoto ${ }^{24}$, A.S. Turcot ${ }^{9}$, M.F. Turner-Watson ${ }^{8}$, P. Utzat ${ }^{11}$, R. Van Kooten ${ }^{12}$, G. Vasseur ${ }^{21}$, P. Vikas ${ }^{18}$, M. Vincter ${ }^{28}$,

A. Wagner ${ }^{27}$, D.L. Wagner ${ }^{9}$, C.P. Ward ${ }^{5}$, D.R. Ward ${ }^{5}$, J.J. Ward ${ }^{15}$, P.M. Watkins ${ }^{1}$, A.T. Watson ${ }^{1}$, N.K. Watson ${ }^{7}$, P. Weber ${ }^{6}$, P.S. Wells ${ }^{8}$, N. Wermes ${ }^{3}$, B. Wilkens ${ }^{10}$, G.W.Wilson ${ }^{27}$, J.A. Wilson ${ }^{1}$, V-H. Winterer ${ }^{10}$, T. Wlodek ${ }^{26}$, G. Wolf ${ }^{26}$, S. Wotton ${ }^{11}$, T.R. Wyatt ${ }^{16}$, A. Yeaman ${ }^{13}$, G. Yekutieli ${ }^{26}$, M. Yurko ${ }^{18}$, V.Zacek ${ }^{18}$, W. Zeuner ${ }^{8}$, G.T. Zorn ${ }^{17}$.

${ }^{1}$ School of Physics and Space Research, University of Birmingham, Birmingham B15 2TT, UK ${ }^{2}$ Dipartimento di Fisica dell' Università di Bologna and INFN, I-40126 Bologna, Italy

${ }^{3}$ Physikalisches Institut, Universität Bonn, D-53115 Bonn, Germany

${ }^{4}$ Department of Physics, University of California, Riverside CA 92521, USA

${ }^{5}$ Cavendish Laboratory, Cambridge CB3 0HE, UK

${ }^{6}$ Carleton University, Department of Physics, Colonel By Drive, Ottawa, Ontario K1S 5B6, Canada

${ }^{7}$ Centre for Research in Particle Physics, Carleton University, Ottawa, Ontario K1S 5B6, Canada

${ }^{8}$ CERN, European Organisation for Particle Physics, CH-1211 Geneva 23, Switzerland

${ }^{9}$ Enrico Fermi Institute and Department of Physics, University of Chicago, Chicago IL 60637, USA

${ }^{10}$ Fakultät für Physik, Albert Ludwigs Universität, D-79104 Freiburg, Germany

${ }^{11}$ Physikalisches Institut, Universität Heidelberg, D-69120 Heidelberg, Germany

${ }^{12}$ Indiana University, Department of Physics, Swain Hall West 117, Bloomington IN 47405, USA

${ }^{13}$ Queen Mary and Westfield College, University of London, London E1 4NS, UK

${ }^{15}$ University College London, London WC1E 6BT, UK

${ }^{16}$ Department of Physics, Schuster Laboratory, The University, Manchester M13 9PL, UK

${ }^{17}$ Department of Physics, University of Maryland, College Park, MD 20742, USA

${ }^{18}$ Laboratoire de Physique Nucléaire, Université de Montréal, Montréal, Quebec H3C 3J7, Canada

${ }^{19}$ University of Oregon, Department of Physics, Eugene OR 97403, USA

${ }^{20}$ Rutherford Appleton Laboratory, Chilton, Didcot, Oxfordshire OX11 0QX, UK

${ }^{21}$ CEA, DAPNIA/SPP, CE-Saclay, F-91191 Gif-sur-Yvette, France

${ }^{22}$ Department of Physics, Technion-Israel Institute of Technology, Haifa 32000, Israel

${ }^{23}$ Department of Physics and Astronomy, Tel Aviv University, Tel Aviv 69978, Israel

${ }^{24}$ International Centre for Elementary Particle Physics and Department of Physics, University of Tokyo, Tokyo 113, and Kobe University, Kobe 657, Japan

${ }^{25}$ Brunel University, Uxbridge, Middlesex UB8 3PH, UK

${ }^{26}$ Particle Physics Department, Weizmann Institute of Science, Rehovot 76100, Israel

${ }^{27}$ Universität Hamburg/DESY, II Institut für Experimental Physik, Notkestrasse 85, D-22607 Hamburg, Germany

${ }^{28}$ University of Victoria, Department of Physics, P O Box 3055, Victoria BC V8W 3P6, Canada

${ }^{29}$ University of British Columbia, Department of Physics, Vancouver BC V6T 1Z1, Canada

${ }^{30}$ University of Alberta, Department of Physics, Edmonton AB T6G 2J1, Canada

${ }^{31}$ Duke University, Dept of Physics, Durham, NC 27708-0305, USA

${ }^{32}$ Technische Hochschule Aachen, III Physikalisches Institut, Sommerfeldstrasse 26-28, D-52056 
Aachen, Germany

${ }^{a}$ Also at TRIUMF, Vancouver, Canada V6T $2 \mathrm{~A} 3$

${ }^{b}$ Royal Society University Research Fellow 


\section{Introduction}

In the Standard Model, a second-order weak transition transforms neutral B mesons into their antiparticles [1]. The neutral B mesons therefore oscillate between particle and antiparticle states before decaying. The frequency of the oscillation depends on the top quark mass, the Cabibbo-Kobayashi-Maskawa matrix elements, and meson decay constants. In analogy to the $K^{0}$ case and neglecting $C P$ violation, the mass eigenstates of $B_{q}^{0}(q=d$ or $s)$ can be described as follows:

$$
\begin{aligned}
& \left|\mathrm{B}_{1}\right\rangle=\frac{1}{\sqrt{2}}\left(\left|\mathrm{~B}_{\mathbf{q}}^{0}\right\rangle+\left|\overline{\mathrm{B}}_{\mathbf{q}}^{0}\right\rangle\right) \\
& \left|\mathrm{B}_{2}\right\rangle=\frac{1}{\sqrt{2}}\left(\left|\mathrm{~B}_{\mathbf{q}}^{0}\right\rangle-\left|\overline{\mathrm{B}}_{\mathbf{q}}^{0}\right\rangle\right)
\end{aligned}
$$

If a $\mathrm{B}_{\mathrm{q}}^{0}$ is produced at time $t=0$, the probabilities of having a $\mathrm{B}_{\mathrm{q}}^{0}$ or a $\overline{\mathrm{B}}_{\mathrm{q}}^{0}$ at proper time $t$ are ${ }^{1}$

$$
\begin{aligned}
& P_{\mathrm{B}_{\mathbf{q}}^{0}}(t)=\frac{1}{\tau} e^{-t / \tau} \cos ^{2}\left(\frac{\Delta m_{\mathbf{q}} t}{2}\right) \\
& P_{\overline{\mathrm{B}}_{\mathbf{q}}^{0}}(t)=\frac{1}{\tau} e^{-t / \tau} \sin ^{2}\left(\frac{\Delta m_{\mathbf{q}} t}{2}\right)
\end{aligned}
$$

where $\tau$ is the $\mathrm{B}_{\mathrm{q}}^{0}$ lifetime. The frequency of the oscillation is given by $\Delta m_{\mathrm{q}}$, the mass difference of the two mass eigenstates $\left(\Delta m_{\mathrm{q}}=m_{\mathrm{B}_{1}}-m_{\mathrm{B}_{2}}\right)$. For $\mathrm{B}_{\mathbf{d}}^{0}-\overline{\mathrm{B}}_{\mathbf{d}}^{0}$ mixing, measurements from ARGUS and CLEO give $x_{\mathrm{d}}=\Delta m_{\mathrm{d}} \tau=0.67 \pm 0.08[2,3]$. Published measurements of the frequency of $B_{d}^{0}-\bar{B}_{d}^{0}$ oscillations made at LEP are available using several different techniques [4, $5,6,7]$.

Extracting information on CKM matrix elements from the measurements of $\Delta m_{\mathrm{d}}$ is prone to large uncertainties due to poorly known meson decay constants. These uncertainties can be reduced by considering the ratio $\Delta m_{\mathrm{s}} / \Delta m_{\mathrm{d}}$. Given the present knowledge of $V_{\mathrm{ts}}$ and $V_{\mathrm{td}}$ one expects $\Delta m_{\mathrm{s}}$ to be of the order of $10 \mathrm{ps}^{-1}$ [8]. The ALEPH collaboration has published a measurement of $\mathrm{B}_{\mathrm{d}}^{0}-\overline{\mathrm{B}}_{\mathrm{d}}^{0}$ oscillations and a lower limit on $\mathrm{B}_{\mathrm{s}}^{0}-\overline{\mathrm{B}}_{\mathrm{s}}^{0}$ oscillations of $\Delta m_{\mathrm{s}}>1.8 \mathrm{ps}^{-1}$ at $95 \%$ confidence level, using events with identified leptons in each thrust hemisphere [7]. This paper describes a similar analysis performed on the OPAL data. In these analyses, leptons are used to tag the presence of $b$ hadron decays and to infer the flavor at decay time. The charge correlation of the two leptons gives information on mixing. The decay time is reconstructed using secondary vertices. Consequently $\mathrm{B}_{\mathrm{d}}^{0}, \mathrm{~B}_{\mathrm{s}}^{0}$ and other $\mathrm{b}$ hadrons all contribute, providing sensitivity to both $\mathrm{B}_{\mathrm{d}}^{0}-\overline{\mathrm{B}}_{\mathrm{d}}^{0}$ and $\mathrm{B}_{\mathrm{s}}^{0}-\overline{\mathrm{B}}_{\mathrm{s}}^{0}$ oscillations.

In this paper, hadronic $\mathrm{Z}^{0}$ decays with two lepton candidates, one in each thrust hemisphere, are selected. The reconstruction of a secondary vertex that includes the lepton is attempted for each lepton candidate, yielding an estimate of the decay length of the $b$ hadron. This is combined with an estimate of the relativistic boost of the $b$ hadron to give the proper decay time. The likelihood of each event is calculated according to the measured proper times and the charge combination of the two leptons. Results on $\Delta m_{\mathrm{d}}$ and $\Delta m_{\mathrm{s}}$ are then obtained using a maximum likelihood technique.

\footnotetext{
${ }^{1}$ The contribution of $\Delta \Gamma$, the difference between the total decay widths of the mass eigenstates, to the oscillations is expected to be negligible and has been ignored.
} 


\section{Event Selection and Simulation}

\subsection{The OPAL Detector and Event Selection}

The OPAL detector has been described elsewhere $[9,10]$. Tracking of charged particles is performed by a central detector, consisting of a silicon microvertex detector, a vertex chamber, a jet chamber and $z$-chambers. ${ }^{2}$ The central detector is positioned inside a solenoid, which provides a uniform magnetic field of $0.435 \mathrm{~T}$. The silicon microvertex detector consists of two layers of silicon strip detectors; the inner layer covers a polar angle range of $|\cos \theta|<0.83$ and the outer layer covers $|\cos \theta|<0.77$. This detector provided both $\phi$ - and $z$-coordinates for data taken in 1993, but $\phi$-coordinates only for 1991 and 1992 . Only $\phi$-coordinate information was used in this analysis. The vertex chamber is a precision drift chamber which covers the range $|\cos \theta|<0.95$. The jet chamber is a large-volume drift chamber, $4 \mathrm{~m}$ long and $3.7 \mathrm{~m}$ in diameter, providing both tracking and $\mathrm{d} E / \mathrm{d} x$ information. The $z$-chambers measure the $z$ coordinate of tracks as they leave the jet chamber in the range $|\cos \theta|<0.72$. The coil is surrounded by a time-of-flight counter array and a lead-glass electromagnetic calorimeter with a presampler. The lead-glass blocks cover the range $|\cos \theta|<0.98$. The magnet return yoke is instrumented with streamer tubes and serves as a hadron calorimeter. Outside the hadron calorimeter are muon chambers, which cover $93 \%$ of the full solid angle.

Hadronic $\mathrm{Z}^{0}$ decays are selected using criteria described in a previous publication [11]. A cone jet algorithm [12] is used to classify tracks and electromagnetic clusters not associated to tracks, into jets. The size of the cone was chosen so as to include nearly all the decay products of $\mathrm{a} b$ hadron into one jet. The jets also include particles produced in the fragmentation process, which originate from the $\mathrm{e}^{+} \mathrm{e}^{-}$collision point. The analysis was performed on data collected in the vicinity of the $\mathrm{Z}^{0}$ peak from 1991 to 1993 . A total of 1539422 hadronic events satisfy the event selection criteria.

\subsection{Event Simulation}

Monte Carlo events are used to predict the relative abundances and decay time distributions for lepton candidates from various physics processes. The JETSET 7.3 Monte Carlo program [13, 14] was used to generate $Z^{0} \rightarrow q \bar{q}$ events which were processed by the detector simulation program [15]. The fragmentation of $b$ and $c$ quarks was parametrised using the fragmentation function of Peterson et al. [16], with $\left\langle x_{E}\right\rangle$ for $\mathrm{b}$ and $\mathrm{c}$ hadrons given by the central values in Table 1.

Standard Model values of the partial widths of the $\mathrm{Z}^{0}$ into $\mathrm{q} \overline{\mathrm{q}}$ were used [17]. The mixture of $c$-flavoured hadrons produced both in $\mathrm{Z}^{0} \rightarrow \mathrm{c} \overline{\mathrm{c}}$ events and in b hadron decays was as prescribed in Reference [18]. The semileptonic branching ratios of charm hadrons and associated uncertainties were also those of Reference [18]. The central values in Table 1 were taken for the inclusive branching ratios for $\mathrm{b} \rightarrow \ell, \mathrm{b} \rightarrow \mathrm{c} \rightarrow \ell$ and $\mathrm{b} \rightarrow \overline{\mathrm{c}} \rightarrow \ell$. The semileptonic branching ratios of the individual $\mathrm{b}$ hadrons were assumed to be proportional to the lifetimes. The models

\footnotetext{
${ }^{2}$ The coordinate system is defined with positive $z$ along the $\mathrm{e}^{-}$beam direction, $\theta$ and $\phi$ being the polar and azimuthal angles. The origin is taken to be the centre of the detector.
} 


\begin{tabular}{|c|c|}
\hline Quantity & Value \\
\hline$\left\langle x_{E}\right\rangle_{b}$ & $0.697 \pm 0.013[19]$ \\
\hline$\left\langle x_{E}\right\rangle_{c}$ & $0.51 \pm 0.02[18]$ \\
\hline$B(\mathrm{~b} \rightarrow \ell)$ & $10.5 \pm 0.6 \pm 0.5 \%[19]$ \\
\hline$B(\mathrm{~b} \rightarrow \mathrm{c} \rightarrow \ell)$ & $7.7 \pm 0.4 \pm 0.7 \%[19]$ \\
\hline$B(\mathrm{~b} \rightarrow \overline{\mathrm{c}} \rightarrow \ell)$ & $1.3 \pm 0.5 \%[13,14,19]$ \\
\hline$M\left(\mathrm{~B}_{\mathrm{s}}^{0}\right)$ & $5.48 \mathrm{GeV}$ \\
\hline$M\left(\Lambda_{\mathrm{b}}\right)$ & $5.62 \mathrm{GeV}$ \\
\hline$\tau_{\mathrm{B}^{+}} / \tau_{\mathrm{B}_{\mathrm{d}}^{0}}$ & $1.01 \pm 0.11[20,21]$ \\
\hline$\tau_{\mathrm{B}_{\mathrm{s}}^{0}} / \tau_{\mathrm{B}_{\mathrm{d}}^{0}}$ & $0.99 \pm 0.18[20,21]$ \\
\hline$\tau_{\Lambda_{\mathrm{b}}} / \tau_{\mathrm{B}_{\mathrm{d}}^{0}}$ & $0.69 \pm 0.12[20,21]$ \\
\hline$\left\langle\tau_{\mathrm{b}}\right\rangle$ & $1.56 \pm 0.02 \mathrm{ps}[20]$ \\
\hline
\end{tabular}

Table 1: The parameters used for the Monte Carlo simulation.

used in describing the semileptonic decays of $b$ and $c$ hadrons were those used in determining the central values in Reference [18]. The generated masses of $\mathrm{B}_{\mathrm{s}}^{0}$ and $\Lambda_{\mathrm{b}}$ particles are also given in Table 1.

The results from LEP [20] and CDF [21] are used in this analysis to determine the lifetimes appropriate to the $\mathrm{b}$ hadron decays, indicated in Table 1 . The values from JETSET for the production fractions of weakly decaying $b$ hadrons were used $[22]$, namely $B^{+}, B_{d}^{0}, B_{s}^{0}, \Lambda_{b}$ in the ratio 39.5:39.5:12:9. The Monte Carlo events used in the bulk of the analysis were generated without mixing. No $\mathrm{B}^{* *}$ nor $\mathrm{D}^{* *}$ production was included in the generation, but the effect of $\mathrm{D}^{* *}$ production was taken into account in the analysis.

\section{Lepton Identification}

Electrons are identified using an artificial neural network [6] which was trained on a sample of Monte Carlo data. Electrons from photon conversions are rejected as in [18]. Muons are identified as in [19]. Lepton candidates are required to satisfy $p>2.0 \mathrm{GeV}$ and $|\cos \theta|<0.9$.

Additional kinematic criteria are imposed to reduce the fraction of leptons in the sample coming from cascade decays of the type $b \rightarrow c \rightarrow \ell$. One expects leptons from primary $b$ decays to be more isolated than leptons from semileptonic charm decays due to the larger $\mathrm{b}$ hadron masses and higher energy neutrinos. To quantify this isolation, the jet containing the lepton candidate is split into two sub-jets, one seeded by the lepton direction, and one remainder jet, where the particles are associatiated with either subjet in an iterative procedure based on their angles to the respective subjet. The energy of the sub-jet containing the lepton candidate, with the energy of the lepton candidate subtracted, is taken as a measure of the isolation. The measured lepton momentum $p$, the lepton momentum transverse to the direction of the jet containing the lepton $\left(p_{t}\right)$, and the isolation measurement are combined using a feedforward neural network to optimize the kinematic selection of leptons from semileptonic $B$ decays. Cutting on this kinematic network output increases the rate of selected direct $\mathrm{b} \rightarrow \ell$ decays relative to cascade decays by $40 \%$ over the relative rate obtained making cuts only on 
combinations of $p$ and $p_{t}$.

If more than one lepton candidate is found in the same thrust hemisphere, ${ }^{3}$ only the candidate with the highest kinematic network output is retained. Events with at least one lepton candidate are classified as inclusive lepton events. Events with at least one lepton candidate in each thrust hemisphere are classified as dilepton events. Dilepton events selected in this manner are known to come primarily from $\mathrm{Z}^{0} \rightarrow \mathrm{b} \overline{\mathrm{b}}$ decays. While the analyses of $\Delta m_{\mathrm{d}}$ and $\Delta m_{\mathrm{s}}$ use only dilepton events, inclusive lepton events are used for systematic checks. Simulated inclusive lepton events are used in parametrising decay time distributions for leptons from different physics processes.

\section{Proper Time Reconstruction}

In reconstructing the proper decay time, each lepton is assumed to be a decay product of $a b$ hadron. The proper time of that hadron is reconstructed using separate estimates of the decay length and the boost. The following sections describe the method.

\subsection{Vertex Reconstruction}

The primary event vertex is reconstructed using the charged tracks in the event along with knowledge of the average position and effective spread of the $\mathrm{e}^{+} \mathrm{e}^{-}$collision point. In this process, tracks that are significantly separated from the vertex position are excluded from the final vertex reconstruction.

The decay point of the $b$ hadron is estimated using a vertex finding algorithm to combine the lepton candidate with other tracks in the same jet. The algorithm assumes these tracks originate either from the interaction point or from the decay point of the $b$ hadron. It tries to select the tracks coming from the $\mathrm{b}$ hadron and forms a common vertex with the lepton track. This approach neglects the fact that some of the tracks come from the decay of a $\mathrm{c}$ hadron, which travels a significant distance before decaying. However, the decay length of the c hadron is usually short relative to that of the $b$ hadron and the opening angle of the c-hadron decay products is on average small compared to the angle between the $\mathrm{c}$ hadron and the lepton. Thus the tracks from the $\mathrm{c}$ hadron extrapolate back close to the b-hadron decay point, and may be combined into a single vertex with the lepton to give a good estimate of this point.

For consideration in the vertex finding algorithm, the lepton candidate track is required to be measured precisely by the vertex detectors, either by the silicon microvertex detector or by the vertex drift chamber. From the other precisely measured tracks in the same jet, seed tracks are chosen and ordered according to the significance of separation from the primary vertex. Seed vertices are formed from the intersection of seed tracks with the lepton track. The algorithm adds tracks which match that vertex better than the primary vertex to form candidate secondary vertices. In $55 \%$ of the cases considered only one candidate vertex is

\footnotetext{
${ }^{3}$ The thrust axis of the event, calculated using charged tracks and electromagnetic clusters unassociated with any charged track, is used to divide the event into two hemispheres.
} 
formed, which is taken as the secondary vertex. In other cases, a single secondary vertex is chosen from the candidates, based on the number of tracks associated to each vertex and the reconstructed vertex positions. The vertex finding is performed in the $x-y$ plane.

The decay length in the $x-y$ plane is calculated from a fit to the estimated primary vertex and secondary vertex positions using the vector momentum sum of the tracks associated to that vertex as a direction constraint, as in the analysis of the decay length of 3-prong $\tau$ decays [23]. In this calculation, the contribution to the error from the uncertainty of the primary vertex position is usually negligible.

Quality requirements are applied to the selected vertices to reduce the contribution from random combinations of tracks, based on the calculated decay length error (from the parameters of the associated tracks), the invariant mass of the associated tracks, the lepton impact parameter and the transverse miss distance. The transverse miss distance is defined as the distance between primary and secondary vertices projected onto the plane orthogonal to the summed momentum vector of the tracks associated to the secondary vertex. In addition, vertices reconstructed with negative decay lengths more than $3 \sigma$ from the primary vertex were

rejected. The net result of all these requirements gives an efficiency of approximately $70 \%$ to reconstruct a secondary vertex starting with an identified lepton from semileptonic b decay in Monte Carlo events. The efficiency is essentially independent of decay length.

The $x-y$ decay length estimate is converted into a 3 -dimensional quantity by dividing by the $\sin \theta$ of the jet axis. The uncertainty in $\sin \theta$ leads to a negligible additional error on the 3-dimensional decay length. A double Gaussian fit to the distribution of the difference between reconstructed and true decay lengths in Monte Carlo $\mathrm{b} \rightarrow \ell$ decays gives $\sigma \sim 400 \mu \mathrm{m}$ for the narrow Gaussian; $67 \%$ of the reconstructed decay lengths lie within $2 \sigma$ of the true values, with $\sigma$ defined by the narrow Gaussian.

\subsection{Boost estimate}

The energy of the parent $b$ hadron is estimated using a novel technique, which assumes that the lepton candidate originates from semileptonic b decay. In outline, using the information contained in the rest of the event and the knowledge of the beam energy, the total energy of the lepton jet (including neutrino) is estimated. The energy in the lepton jet due to fragmentation particles, originating from the primary vertex, is then estimated by classifying tracks and clusters as either $b$ decay products or fragmentation particles using kinematics and impact parameters. The energy of the parent $b$ hadron is given simply by subtracting the fragmentation energy from the total jet energy.

The total energy of the jet (including the neutrino) is estimated by treating the whole event as a two-body decay of an object of mass $M$. The two bodies have masses $m_{1}$ and $m_{2}$, and energies $E_{1}$ and $E_{2}$. By energy and momentum conservation,

$$
E_{1}=\frac{M^{2}+m_{1}^{2}-m_{2}^{2}}{2 M} .
$$

One of the bodies is taken to be the lepton jet including the neutrino, and the other to be the rest of the event. The mass of the jet containing the lepton is approximated to be the $B_{d}^{0}$ mass, 
$5.28 \mathrm{GeV}$ (the result is insensitive to this assumption), and $M$ is taken to be the $\mathrm{Z}^{0}$ mass. The mass of the rest of the event, $m_{2}$, is calculated by summing over those tracks and unassociated clusters which are not included in the lepton jet, where the charged particles are assumed to be pions. The value of $m_{2}$ is scaled according to the inverse of the total reconstructed energy in the event in order to improve the resolution.

Tracks in the lepton jet are classified either as fragmentation products or as decay products of the inferred $b$ hadron based on three pieces of information: the probability of the track to extrapolate back to the primary vertex relative to the secondary vertex, the track momentum, and the angle of the track relative to the estimated direction of the c-hadron resulting from the semileptonic b decay. This direction is estimated from the tracks associated to the secondary vertex excluding the lepton candidate. Since the mass of the $\mathrm{D}$ is relatively small, the decay products are relatively collimated about the D direction, although the angles are highly correlated with the track momenta through simple kinematics. The relative probability for a track of a given momentum and angle to be from fragmentation or a D decay product, is taken from Monte Carlo. This probability is then combined with that calculated from the vertex information as independent quantities and used to assign a weight for each track. The separating power between decay products and fragmentation products improves significantly with decay length due to the use of vertex information.

The neutral fragmentation energy is more difficult to identify. No vertex information is available, and no attempt is made to use the cluster energies to make the distinction. Only the angle of the unassociated electromagnetic clusters relative to the estimated D direction is used to assign the weight for each cluster.

The estimated b hadron energy, $E_{B}$, is taken to be

$$
E_{B}=E_{1}-E_{\text {frag }}
$$

where $E_{\text {frag }}$ is the (weighted) sum of charged and neutral energy of fragmentation products in the lepton jet. The overall resolution of the boost estimate improves with decay length, and degrades as the fragmentation energy subtracted increases. For example, for decay lengths longer than $0.2 \mathrm{~cm}$ in the $x-y$ plane, the standard deviation is about $12 \%$ and the central Gaussian of a double Gaussian fit has a width of about 7\%. No significant bias in the estimated boost is observed in Monte Carlo between inclusive leptons and leptons in dilepton events, where the extra neutrino could cause problems for the total reconstructed energy.

\subsection{Proper time estimate}

The proper time, $t$ is formed from the (3-dimensional) decay length $L$ and boost:

$$
t=\frac{L}{\beta \gamma}=\frac{m_{B}}{\sqrt{E_{B}^{2}-m_{B}^{2}}} \cdot L
$$

The distribution of this estimate vs. the true proper time is shown in Figure 1 for Monte Carlo semileptonic b decays. In order to see the resolution more clearly, Figure 2 shows projections of $t$ and $t-t^{\prime}$, where $t^{\prime}$ is the true proper time, in three slices of $t^{\prime}$. 
The measured proper time deviates from the true value due to measurement errors in the decay length of the reconstructed secondary vertex and in the Lorentz boost factor. The assignment of particles to the secondary vertex is imperfect, resulting in long tails on the measurement deviation, and in a small fraction of the vertices being misreconstructed near the primary vertex even when the true decay length is large. These effects are evident in Figures 1 and 2. The contribution of the decay length measurement to the overall proper time resolution is on average slightly larger than the contribution from the uncertainty in the boost factor at all decay lengths. A double Gaussian fit to the distribution of the deviation between the measured proper time and the true time, for primary $\mathrm{b} \rightarrow \ell$ decays in the Monte Carlo, gives $\sigma=0.26 \mathrm{ps}$ for the narrow Gaussian. Of the measured proper times, $68 \%$ lie within $2 \sigma$ of the true times. The distribution of $t-t^{\prime}$ has a complicated shape and depends on the true time itself. A Monte Carlo simulation is therefore used to parametrise the proper time resolution.

\section{$5 \quad$ Likelihood Function}

In order to construct the likelihood function, the decay proper time probability density functions (p.d.f.) for like-sign and unlike-sign leptons are estimated and parametrised using Monte Carlo data without $\mathrm{B}-\overline{\mathrm{B}}$ mixing. The sources of lepton candidates $(\ell)$ are grouped according to their decay time distributions and their charge correlations with the decaying $b$ quark. Sources of lepton candidates (including misidentified hadrons) in the absence of $\mathrm{B}-\overline{\mathrm{B}}$ mixing are defined as follows (charge conjugate processes are implied):

(1) Lepton candidates in light quark pair events ( $u \bar{u}, \mathrm{~d} \overline{\mathrm{d}}$ and $s \bar{s})$,

$(2) \mathrm{c} \rightarrow \ell^{+}$in $\mathrm{Z}^{0} \rightarrow \mathrm{c} \overline{\mathrm{c}}$ events,

(3) Lepton candidates in $\mathrm{Z}^{0} \rightarrow \mathrm{c} \overline{\mathrm{c}}$ events which are not included in (2),

(4) $\mathrm{b} \rightarrow \overline{\mathrm{B}}_{\mathrm{d}}^{0} \rightarrow \ell^{+}$(mainly from $\mathrm{b} \rightarrow \mathrm{c} \rightarrow \ell^{+} \nu \mathrm{X}$ ),

$(5) \mathrm{b} \rightarrow \overline{\mathrm{B}}_{\mathrm{s}}^{0} \rightarrow \ell^{+}$(mainly from $\mathrm{b} \rightarrow \mathrm{c} \rightarrow \ell^{+} \nu \mathrm{X}$ ),

$(6) \mathrm{b} \rightarrow\left(\mathrm{B}^{-}\right.$or $\left.\Lambda_{\mathrm{b}}\right) \rightarrow \ell^{+}$(mainly from $\left.\mathrm{b} \rightarrow \mathrm{c} \rightarrow \ell^{+} \nu \mathrm{X}\right)$,

(7) $\mathrm{b} \rightarrow \overline{\mathrm{B}}_{\mathrm{d}}^{0} \rightarrow \ell^{-}$(mainly from $\mathrm{b} \rightarrow \ell^{-} \bar{\nu} \mathrm{X}$ ),

(8) $\mathrm{b} \rightarrow \overline{\mathrm{B}}_{\mathrm{s}}^{0} \rightarrow \ell^{-}$(mainly from $\mathrm{b} \rightarrow \ell^{-} \bar{\nu} \mathrm{X}$ ),

$(9) \mathrm{b} \rightarrow\left(\mathrm{B}^{-}\right.$or $\left.\Lambda_{\mathrm{b}}\right) \rightarrow \ell^{-}$(mainly from $\left.\mathrm{b} \rightarrow \ell^{-} \bar{\nu} \mathrm{X}\right)$,

(10) Lepton candidates in $Z^{0} \rightarrow b \bar{b}$ events not coming from long-lived $b$ hadrons.

Candidates from categories (1), (3) and (10) have only a small charge correlation with the primary quark, which is taken into account.

The fractions of leptons in inclusive lepton events and dilepton events coming from $\mathrm{Z}^{0} \rightarrow \mathrm{b} \overline{\mathrm{b}}$, $\mathrm{Z}^{0} \rightarrow \mathrm{c} \overline{\mathrm{c}}$, and $\mathrm{Z}^{0}$ decays to light quark pairs are given in Table 2. Also shown are the relative 
probabilities for leptons from different sources for each type of $Z^{0} \rightarrow q \bar{q}$ decay as estimated from the Monte Carlo simulation. For lepton candidates in $\mathrm{Z}^{0} \rightarrow \mathrm{b} \overline{\mathrm{b}}$ events, the relative probabilities of each of the categories (4)-(10) are denoted by $g_{4}-g_{10}$; the sum of $g_{4}$ through $g_{10}$ is unity. Similarly for lepton candidates in $\mathrm{Z}^{0} \rightarrow \mathrm{c} \overline{\mathrm{c}}$ events, the relative probabilities of categories (2) and (3) are denoted by $g_{2}$ and $g_{3}$, with $g_{2}+g_{3}=1$. As a shorthand, we refer to $\left(g_{4}+g_{5}+g_{6}\right)$ as the cascade fraction.

\begin{tabular}{|c||l||l|c|}
\hline primary flavour & $\begin{array}{l}\text { inclusive lepton } \\
\text { fraction }\end{array}$ & $\begin{array}{l}\text { dilepton } \\
\text { fraction }\end{array}$ & $\begin{array}{c}\text { relative amount } \\
\text { per source }\end{array}$ \\
\hline $\mathrm{u} \overline{\mathrm{u}}, \mathrm{d} \overline{\mathrm{d}}$, or $\mathrm{s} \overline{\mathrm{s}}$ & $r_{\mathrm{uds}}=\mathbf{0 . 0 8 9}$ & $r_{\mathrm{uu} \overline{\mathrm{d} s} \overline{\mathrm{s}}}=\mathbf{0 . 0 0 9}$ & $g_{1}=1.000$ \\
\hline $\mathrm{c} \overline{\mathrm{c}}$ & $r_{\mathrm{c}}=\mathbf{0 . 0 9 6}$ & $r_{\mathrm{c} \overline{\mathbf{c}}}=\mathbf{0 . 0 2 9}$ & $g_{2}=0.784$ \\
& & & $g_{3}=0.216$ \\
\hline $\mathrm{b} \overline{\mathrm{b}}$ & $r_{\mathrm{b}=0.815}$ & $r_{\mathrm{b} \overline{\mathrm{b}}=0.962}$ & $g_{4}=0.041$ \\
& & & $g_{5}=0.006$ \\
& & & $g_{6}=0.027$ \\
& & & $g_{7}=0.350$ \\
& & & $g_{8}=0.112$ \\
& & & $g_{9}=0.454$ \\
& & & $g_{10}=0.010$ \\
\hline
\end{tabular}

Table 2: The fractional compositions of leptons in inclusive lepton events $\left(r_{q}\right)$ and dilepton events $\left(r_{\mathrm{q} \overline{\mathrm{q}}}\right)$ for $\mathrm{b} \overline{\mathrm{b}}, \mathrm{c} \overline{\mathrm{c}}$, and light quark events. The $g_{i}$ 's are the number of thrust hemispheres with a lepton candidate from category $i$ normalized to the number of thrust hemispheres with a lepton from the same quark flavour class. The sum of the $g_{i}$ for each flavour category is unity.

The parametrisation of the p.d.f. for each component is described below. In the absence of B mixing the parametrisation for category $i(i=1, \ldots, 10)$ is described by the general form

$$
f_{i}(t)=\int_{0}^{\infty} d t^{\prime} \frac{1}{\tau_{i}} e^{-\frac{t^{\prime}}{\tau_{i}}}\left\{b_{i} u_{i}(t)+\left(1-b_{i}\right) v_{i}\left(t^{\prime}-t\right)\right\},
$$

where $t$ is the measured time, $t^{\prime}$ is the true time and $\int_{-\infty}^{+\infty} f_{i}(t) d t=1$. The $e^{-t^{\prime} / \tau_{i}}$ term describes the lifetime distribution for vertices originating from weak hadron decays. The function $v_{i}$ is the resolution function for the bulk of the vertices, and the function $u_{i}$ describes the subset of vertices which are misreconstructed near the primary vertex. Since the proper time resolution is found to degrade linearly with $t^{\prime}$ in the Monte Carlo, the resolution parameters in the functions $u_{i}$ and $v_{i}$ are linear functions of $t^{\prime}$. The same functions $u_{i}, v_{i}$ and parameters $b_{i}$ are used for lepton candidates in categories (7)-(9), and differ slightly from those used for categories (4)-(6) (mainly from cascade decays). The parameters in the p.d.f. are fixed using a large statistics Monte Carlo sample of events with at least one lepton candidate for which a secondary vertex has been found. The $\tau_{i}$ for categories (1)-(3) and (10) are taken from the Monte Carlo simulation; the values for categories (4)-(9) are obtained from experimental data cited in Table 1.

Except for the obvious primary flavour correlation between the two thrust hemispheres, we assume that the production probability of a $B_{d}^{0}$ in one hemisphere is independent of producing $\overline{\mathrm{B}}_{\mathrm{d}}^{0}, \mathrm{~B}^{-}, \overline{\mathrm{B}}_{\mathrm{s}}^{0}$ or $\mathrm{b}$ baryons in the other hemisphere. With this assumption, the rate of events 
with a lepton of category $i$ in one thrust hemisphere and $j$ in the other hemisphere is calculated relative to the total rate of dilepton events for like-sign events, $\xi_{i j}$, and for unlike-sign lepton events, $\eta_{i j}$. The parameters $\xi_{i j}$ and $\eta_{i j}$ are calculated from the numbers in Table 2 and are zero for the cases where $i$ and $j$ have different primary flavour. For example, in the case where one of the leptons is from cascade decay $(i=4,5,6)$ and the other is from direct decay $(j=7,8,9)$, $\xi_{i j}=\xi_{j i}=g_{i} g_{j} r_{\mathrm{bb}}$ and $\eta_{i j}=0$.

To account for $\mathrm{B}$ mixing in the above formulation, the functions $f_{i}$ are modified for categories $(4),(5),(7)$ and (8) to keep the charge correlation the same in each category. For example, decays of the type $\mathrm{b} \rightarrow \overline{\mathrm{B}}_{\mathrm{d}}^{0} \rightarrow \mathrm{B}_{\mathrm{d}}^{0} \rightarrow \ell^{+} \nu \mathrm{X}$ are included in category (4). The proper time function for $\overline{\mathrm{B}}_{\mathrm{d}}^{0} \rightarrow \ell^{+}, f_{4}(t)$, is modified to $\tilde{f}_{4}(t)$, where

$$
\begin{aligned}
g_{4} \tilde{f}_{4}(t) & =g_{4} \int_{0}^{\infty} d t^{\prime} \frac{1}{\tau_{4}} e^{-\frac{t^{\prime}}{\tau_{4}}}\left\{\left(1-\chi_{\mathrm{d}}\right) b_{4} u_{4}(t)+\left(1-b_{4}\right) v_{4}\left(t^{\prime}-t\right) \cos ^{2}\left(\frac{\Delta m_{\mathrm{d}} t^{\prime}}{2}\right)\right\} \\
& +g_{7} \int_{0}^{\infty} d t^{\prime} \frac{1}{\tau_{7}} e^{-\frac{t^{\prime}}{\tau_{7}}}\left\{\chi_{\mathrm{d}} \quad b_{7} u_{7}(t)+\left(1-b_{7}\right) v_{7}\left(t^{\prime}-t\right) \sin ^{2}\left(\frac{\Delta m_{\mathrm{d}} t^{\prime}}{2}\right)\right\}
\end{aligned}
$$

where the second line comes from the mixing of $\bar{B}_{\mathbf{d}}^{0} \rightarrow \mathrm{B}_{\mathbf{d}}^{0} \rightarrow \ell^{+}$, and $\chi_{\mathbf{d}}=x_{\mathbf{d}}^{2} /\left(2+2 x_{\mathbf{d}}^{2}\right)$. Similarly the proper time functions $\tilde{f}_{5}, \tilde{f}_{7}$ and $\tilde{f}_{8}$ are constructed by including the mixing effects. For all other categories $\tilde{f}_{i}=f_{i}$. The parameters $\xi_{i j}$ and $\eta_{i j}$ are not modified with the introduction of mixing.

The overall probability density function for like-sign dilepton events with two secondary vertices is constructed from the parametrised decay proper time distribution function $\left(\tilde{f}_{i}\right)$ of each category and the relative rate of the possible combination of two categories $\left(\xi_{i j}\right)$ :

$$
F_{2}\left(t_{1}, t_{2}\right)=\sum_{i=1}^{10} \sum_{j=1}^{10} \xi_{i j} \tilde{f}_{i}\left(t_{1}\right) \tilde{f}_{j}\left(t_{2}\right)
$$

Similarly, for unlike-sign dilepton events,

$$
G_{2}\left(t_{1}, t_{2}\right)=\sum_{i=1}^{10} \sum_{j=1}^{10} \eta_{i j} \tilde{f}_{i}\left(t_{1}\right) \tilde{f}_{j}\left(t_{2}\right)
$$

The normalization ensures that

$$
\sum_{i=1}^{10} \sum_{j=1}^{10}\left(\xi_{i j}+\eta_{i j}\right) \int_{-\infty}^{+\infty} d t_{1} \tilde{f}_{i}\left(t_{1}\right) \int_{-\infty}^{+\infty} d t_{2} \tilde{f}_{j}\left(t_{2}\right)=1
$$

When only one secondary vertex is found, the overall probability density functions $F_{1}\left(t_{1}\right)$ and $G_{1}\left(t_{1}\right)$ can be derived by integrating over $t_{2}$ from $-\infty$ to $+\infty$.

The total likelihood function is given by

$$
\mathcal{L}\left(\Delta m_{\mathbf{d}}, \ldots\right)=\prod_{i=1}^{N_{1}^{L S}} F_{1}\left(t_{1 i}\right) \prod_{j=1}^{N_{1}^{U S}} G_{1}\left(t_{1 j}\right) \prod_{m=1}^{N_{2}^{L S}} F_{2}\left(t_{1 m}, t_{2 m}\right) \prod_{n=1}^{N_{2}^{U S}} G_{2}\left(t_{1 n}, t_{2 n}\right),
$$

where $N_{1}^{L S}\left(N_{1}^{U S}\right)$ is the number of like-sign (unlike-sign) dilepton events with one secondary vertex reconstructed and $N_{2}^{L S}\left(N_{2}^{U S}\right)$ is the number of like-sign (unlike-sign) events with two vertices reconstructed. 


\section{$6 \quad$ Fit Results for $\Delta m_{\mathrm{d}}$}

The numbers of dilepton events with at least one secondary vertex constructed for the combination of e-e, e- $\mu$ and $\mu-\mu$, are listed in Table 3, separately for like-sign and unlike-sign dilepton events. Also included is the total number of secondary vertices reconstructed in these events.

\begin{tabular}{|l||c|c|c||c|c|}
\hline & $\mathrm{e}-\mathrm{e}$ & $\mathrm{e}-\mu$ & $\mu-\mu$ & total & total vertices \\
\hline \hline unlike-sign & 446 & 978 & 620 & 2044 & 3261 \\
\hline like-sign & 206 & 426 & 289 & 921 & 1460 \\
\hline
\end{tabular}

Table 3: The numbers of dilepton events with at least one secondary vertex reconstructed for the combinations $\mathrm{e}-\mathrm{e}, \mathrm{e}-\mu$ and $\mu-\mu$, separately for like-sign and unlike-sign leptons. Also indicated is the total number of secondary vertices reconstructed in unlike-sign and like-sign dilepton events.

Figure 3 shows the distribution of decay times for all leptons in the dilepton sample and separately for leptons in like-sign and unlike-sign events. The curves are the results of the likelihood fit described below.

In Figure $4, \ln \left(\mathcal{L}_{\max } / \mathcal{L}\right)$ is plotted as a function of $\Delta m_{\mathrm{d}}$ and $\Delta m_{\mathrm{s}}$ with all other parameters at their the nominal values, where $\mathcal{L}_{\max }$ is the maximum value of the likelihood function. The contour lines are at $\ln \left(\mathcal{L}_{\max } / \mathcal{L}\right)$ values corresponding to $1 \sigma, 2 \sigma, 3 \sigma$, etc. For $\Delta m_{\mathrm{s}}>2 \mathrm{ps}^{-1} \Delta m_{\mathrm{d}}$ and $\Delta m_{\mathrm{s}}$ are almost completely decoupled.

To determine $\Delta m_{\mathbf{d}}$ a three parameter fit is performed, varying $\Delta m_{\mathbf{d}}$ simultaneously with the cascade fraction and the $\mathrm{B}_{\mathrm{s}}^{0}$ fraction. Gaussian constraints with the proper systematic errors are imposed on these two parameters. The relative uncertainty in the cascade fraction is taken to be $\pm 15 \%$ [18], which includes uncertainties due to branching fractions, decay modelling and detector simulation. The relative uncertainty in the $\mathrm{B}_{\mathrm{s}}^{0}$ production rate is taken to be $\pm 30 \%$. Our knowledge of this rate is discussed in the Appendix. In the fit the $\mathrm{B}_{\mathrm{s}}^{0}$ oscillation parameter is fixed at $\Delta m_{\mathrm{s}}=10.0 \mathrm{ps}^{-1}$. The result of the fit is $\Delta m_{\mathrm{d}}=0.462 \pm 0.057 \mathrm{ps}^{-1}$. The fitted value of the cascade fraction is $0.078 \pm 0.010$ (the nominal value is 0.074 ), and the fitted value of $B\left(\mathrm{~b} \rightarrow \overline{\mathrm{B}}_{\mathrm{s}}^{0}\right)$ is $0.148 \pm 0.024$ (the nominal value is 0.120 ).

The fraction of like-sign leptons as a function of proper decay time

$$
\mathcal{R}(t)=\frac{N^{L S}(t)}{N^{U S}(t)+N^{L S}(t)}
$$

is plotted in Figure 5 for data. In the figure, the expected curve for $\Delta m_{\mathrm{d}}=0.462 \mathrm{ps}^{-1}$ is shown as the solid line, while the dashed line shows the expected curve with no $B_{d}^{0}$ oscillations. The fitted values of the cascade fraction and the fraction of leptons from $\mathrm{B}_{\mathrm{s}}^{0}$ decays are used for the solid line. The same values of these parameters were used for the dashed line. Events in which vertices have been reconstructed in both thrust hemispheres enter the plot twice. Note that the rise in $\mathcal{R}$ at negative values of $t$ is due to background leptons. 


\section{$7 \quad$ Systematic Errors on $\Delta m_{\mathrm{d}}$}

In the three parameter fit, the error on $\Delta m_{\mathrm{d}}$ is a combination of statistical error and systematic error due to the constraints on the cascade decay fraction and the $\mathrm{B}_{\mathrm{s}}^{0}$ fraction. The systematic error from the cascade decay fraction is estimated by repeating the fit with the central value of the cascade fraction changed by $+15 \%$ or $-15 \%$ (the systematic uncertainty on this parameter) from its nominal value. The systematic error resulting from the $B_{s}^{0}$ fraction is obtained in a similar way. The statistical error on $\Delta m_{\mathrm{d}}$ is ${ }_{-0.053}^{+0.040} \mathrm{ps}^{-1}$, obtained by subtracting in quadrature these two systematic errors from the fitting error.

The uncertainty due to the resolution function description was assessed by repeating the parametrisation using Monte Carlo events in which the tracking resolution was degraded by $14 \%$ [24]. The exercise was repeated with the resolution improved by $14 \%$. The uncertainty in the background from $\mathrm{Z}^{0} \rightarrow \mathrm{c} \overline{\mathrm{c}}$ events is taken to be $\pm 30 \%$ due to uncertainties in the branching fractions and modelling of semileptonic charm decays, the relative production rates of charmed hadrons, and the uncertainty in the partial width for $Z^{0} \rightarrow c \bar{c}$. The production rates of $B_{d}^{0}$ and $\mathrm{B}^{+}$are assumed to be equal and the b-baryon production fraction is assumed to lie in the range $0.09 \pm 0.04$. The decay of $B$ mesons to $\mathrm{D}^{* *}$ mesons, not included in the Monte Carlo, affects the relative rate of cascade decays originating from the different $B$ mesons. The systematic error was estimated by simulating the effect of $40 \% \mathrm{D}^{* *}$ production in $\mathrm{B}$ meson decays. Variations in the efficiency of the secondary vertex reconstruction as a function of decay length are found to have a negligible effect on $\Delta m_{\mathrm{d}}$. Uncertainties in the source composition due to Monte Carlo statistics have been neglected.

The B lifetime variations were performed by changing the ratios of individual B lifetimes while keeping the average lifetime fixed at the LEP average value, $1.56 \mathrm{ps}^{-1}$. A one parameter fit to the average $B$ lifetime in the inclusive lepton sample yields $1.58 \pm 0.01 \mathrm{ps}$ (statistical error only). This result is consistent with the LEP average value.

The summary of the sources and estimated values of systematic errors are listed in Table 4. The sum of these systematic errors in quadrature is $\delta \Delta m_{\mathbf{d}}={ }_{-0.035}^{+0.052} \mathrm{ps}^{-1}$.

\section{Fit results for $\Delta m_{\mathrm{s}}$}

We use the dependence of the likelihood on the assumed value of $\Delta m_{\mathrm{s}}$ to constrain the true value of $\Delta m_{\mathrm{s}}$. As can be seen in Figure 4, this dependence does not allow us to derive an upper bound on $\Delta m_{\mathrm{s}}$; therefore we derive only a lower limit. First we consider the case where systematic errors are ignored, and then we show how the limit is modified when these errors are considered.

In the absence of systematic errors, we set the limit simply on the basis of the difference in log-likelihood with respect to the maximum value. To avoid a possible bias due to the correlation of $\Delta m_{\mathrm{d}}$ with $\Delta m_{\mathrm{s}}$ at low values of $\Delta m_{\mathrm{s}}$, the value of $\Delta m_{\mathrm{d}}$ is taken from an average of LEP measurements which are not themselves sensitive to $\Delta m_{\mathrm{s}}$ (i.e. excluding dilepton results), $\Delta m_{\mathbf{d}}=0.52 \pm 0.05 \mathrm{ps}^{-1}[4,5,6]$. Fixing $\Delta m_{\mathbf{d}}$ to $0.52 \mathrm{ps}^{-1}$, all $\Delta m_{\mathrm{s}}$ values giving a log-likelihood difference larger than 1.92 can be excluded at $95 \%$ confidence level. Defined this way, for any 


\begin{tabular}{|c|c|}
\hline Source of uncertainty & $\delta \Delta m_{\mathrm{d}} \mathrm{ps}^{-1}$ \\
\hline \hline cascade decay fraction $( \pm 15 \%)$ & ${ }_{-0.007}^{+0.015}$ \\
\hline $\mathrm{B}_{\mathrm{s}}^{0}$ fraction $( \pm 30 \%)$ & ${ }_{-0.018}^{+0.039}$ \\
\hline resolution function $( \pm 14 \%)$ & ${ }_{-0.002}^{+0.003}$ \\
\hline lepton misidentification $(\mathrm{e}: \pm 30 \%, \mu: \pm 20 \%)$ & ${ }_{ \pm 0.011}$ \\
\hline charm background $( \pm 30 \%)$ & ${ }_{-0.004}^{+0.005}$ \\
\hline b-baryon fraction $( \pm 0.04)$ & ${ }_{-0.011}^{+0.013}$ \\
\hline $\mathrm{b} \rightarrow \mathrm{D}^{* *}$ decays & -0.008 \\
\hline$\tau_{\mathrm{B}^{+}} / \tau_{\mathrm{B}_{\mathrm{d}}^{0}}=1.01 \pm 0.11$ & ${ }_{-0.023}^{+0.020}$ \\
$\tau_{\mathrm{B}_{\mathrm{s}}^{0}} / \tau_{\mathrm{B}_{\mathrm{d}}^{0}}=0.99 \pm 0.18$ & ${ }_{-0.003}^{+0.005}$ \\
$\tau_{\Lambda_{\mathrm{b}}} / \tau_{\mathrm{B}_{\mathrm{d}}^{0}}=0.69 \pm 0.12$ & ${ }_{-0.003}^{+0.004}$ \\
\hline$\Delta m_{\mathrm{s}}=2-20 \mathrm{ps}^{-1}$ & ${ }_{-0.000}^{+0.012}$ \\
\hline \hline Total systematic error & ${ }_{-0.035}^{+0.052}$ \\
\hline
\end{tabular}

Table 4: Summary of systematic error estimations for $\Delta m_{\mathrm{d}}$ measurement.

excluded value of $\Delta m_{\mathrm{s}}, 95 \%$ of experiments would give a log-likelihood difference smaller than that observed, if it were the true value. Using this technique we find $\Delta m_{\mathrm{s}}>2.3 \mathrm{ps}^{-1}$ at $95 \%$ confidence level. The difference in log-likelihood from the point of maximum likelihood as a function of $\Delta m_{\mathrm{s}}$ is shown as the dashed curve in Figure 6.

To account for systematic errors in setting a limit, we maximize the log-likelihood with respect to the values of all parameters, constrained by their systematic uncertainties, at each value of $\Delta m_{\mathrm{s}}$. Values of $\Delta m_{\mathrm{s}}$ where the resulting log-likelihood differs by more than 1.92 from the maximum value of log-likelihood, over the full $\Delta m_{\mathrm{s}}$ range, are excluded as before. This technique of including the systematic errors has the advantage that the confidence level quoted is accurate irrespective of whether statistical or systematic errors dominate. Furthermore, the effect of the systematic uncertainties is assessed using data. The constraints are those shown in Table 4 . In addition, $\Delta m_{\mathbf{d}}$ is treated as a systematic uncertainty constrained by the LEP average given above. The incorporation of systematic errors reduces the limit from 2.3 to 2.2 $\mathrm{ps}^{-1}$. The solid curve in Figure 6 shows the difference in log-likelihood from the maximum as a function of $\Delta m_{\mathrm{s}}$ with systematic errors included.

\section{$9 \quad$ Study of $\Delta m_{\mathrm{s}}$ analysis using simulated events}

In order to check the analysis technique and to study the expected sensitivity to $\Delta m_{\mathrm{s}}$, a set of Monte Carlo experiments were performed using a fast simulation. In these tests the decay time distributions and the populations of the different lepton categories were generated using the same functions and values used in the likelihood function. The statistical behaviour of the fit was studied by generating 200 independent Monte Carlo datasets with the same numbers of events as the data at 4 sets of input parameters $\left(\Delta m_{\mathrm{d}}=0.5 \mathrm{ps}^{-1}\right.$ and $\Delta m_{\mathrm{s}}=1.0,2.0,4.0$ and $\left.15.0 \mathrm{ps}^{-1}\right)$. The results of these studies are shown in Figure 7. Each row corresponds to a different generated value of $\Delta m_{\mathrm{s}}$, indicated by the $\Delta m_{\mathrm{s}}^{*}$ at the right edge of the plot. The first column shows the fitted value of $\Delta m_{\mathrm{s}}$ for each trial. In the second column are distributions 
of the log-likelihood difference between the value at the fitted maximum and the value at the generated $\Delta m_{\mathrm{s}}$ for each trial. The number of trials giving a log-likelihood difference of more than 1.92 units are consistent with the expected $5 \%$. The third column shows where one could expect to set the lower limit on $\Delta m_{\mathrm{s}}$ at $95 \%$ confidence level for each trial, based purely on statistics. The limit quoted in the previous section is consistent with these distributions generated for $\Delta m_{\mathrm{s}}$ larger than $2 \mathrm{ps}^{-1}$.

A sample of approximately 2 million fully simulated hadronic $\mathrm{Z}^{0}$ decays was also analyzed. The charges of leptons coming from $B_{d}^{0}$ and $B_{s}^{0}$ decays were reversed as a function of the true proper decay time to simulate $\Delta m_{\mathrm{d}}=0.5 \mathrm{ps}^{-1}$ and a variety of $\Delta m_{\mathrm{s}}$ values: $\Delta m_{\mathrm{s}}=1.0,2.0,4.0$, and $15.0 \mathrm{ps}^{-1}$. In each case the maximum log-likelihood differs by less than 1.92 from the loglikelihood at the generated value. Note that the trials are not statistically independent.

\section{Conclusion}

We have measured the oscillation frequency $\Delta m_{\mathrm{d}}$ by measuring the proper time of $\mathrm{B}$ meson decays and tagging the charges of leptons in both thrust hemispheres. The $\mathrm{B}_{\mathbf{d}}^{0}-\overline{\mathrm{B}}_{\mathbf{d}}^{0}$ oscillation parameter is measured to be

$$
\Delta m_{\mathbf{d}}=0.462_{-0.053}^{+0.040}{ }_{-0.035}^{+0.052} \mathrm{ps}^{-1}
$$

corresponding to $\left(3.04{ }_{-0.35}^{+0.26}{ }_{-0.22}^{+0.34}\right) \times 10^{-4} \mathrm{eV}$.

The $\Delta m_{\mathrm{d}}$ value is consistent with the previous OPAL measurements $[5,6]$ of $\Delta m_{\mathrm{d}}=$ $0.508 \pm 0.075 \pm 0.025 \mathrm{ps}^{-1}$ using $\mathrm{B}_{\mathbf{d}}^{0} \rightarrow \mathrm{D}^{* \pm} \ell^{\mp} X$ together with jet charge, and $\Delta m_{\mathbf{d}}=0.57 \pm$ $0.11 \pm 0.02 \mathrm{ps}^{-1}$ using $\mathrm{D}^{* \pm}$ and leptons in opposite hemispheres. These results have very little statistical overlap. In addition, the dominant systematic errors of each method differ. Ignoring the tiny statistical correlations and accounting for small correlations in the systematic errors, we find

$$
\Delta m_{\mathrm{d}}=0.496 \pm 0.046 \mathrm{ps}^{-1} .
$$

Using $\tau_{\mathrm{B}_{\mathrm{d}}^{0}}=1.50 \pm 0.10 \mathrm{ps}$, the combined OPAL value gives $x_{\mathrm{d}}=0.74 \pm 0.07 \pm 0.05$, where the last error is due to the uncertainty in $\tau_{\mathrm{B}_{\mathrm{d}}^{0}}$. This value is also consistent with the average of ARGUS and CLEO measurements, $x_{\mathbf{d}}=0.67 \pm 0.08[2,3]$.

We derive the limit

$$
\Delta m_{\mathrm{s}}>2.2 \mathrm{ps}^{-1}
$$

at $95 \%$ confidence level. If we assume $\Delta m_{\mathrm{s}} / \Delta m_{\mathrm{d}}=(1.35 \pm 0.15)\left|V_{\mathrm{ts}} / V_{\mathrm{td}}\right|^{2}[8]$, we obtain $\left|V_{\mathrm{ts}} / V_{\mathrm{td}}\right|>1.72$ at $95 \%$ confidence level, where the limit is obtained in a similar fashion to that described in Section 8, treating the factor $1.35 \pm 0.15$ as an additional systematic error. 


\section{Appendix : The rate of $\overline{\mathrm{B}}_{\mathrm{s}}^{0}$ production}

Using the value of $B\left(\mathrm{D}_{\mathrm{s}} \rightarrow \phi \pi^{+}\right)$in reference $[3]$ we derive $B\left(\mathrm{~b} \rightarrow \overline{\mathrm{B}}_{\mathrm{s}}^{0} \rightarrow \mathrm{D}_{\mathrm{s}} \ell^{-} \bar{\nu} \mathrm{X}\right)=1.33 \pm 0.36 \%$ from the product branching ratios quoted in reference [25]. The branching fraction for $\overline{\mathrm{B}}_{\mathrm{s}}^{0} \rightarrow$ $\mathrm{D}_{\mathrm{s}} \ell^{-} \bar{\nu} \mathrm{X}$ is likely to be less than $\mathrm{B}\left(\overline{\mathrm{B}}_{\mathrm{s}}^{0} \rightarrow \ell^{-} \bar{\nu} \mathrm{X}\right)$ due to the presence of decays of the type $\overline{\mathrm{B}}_{\mathrm{s}}^{0} \rightarrow \mathrm{D}_{\mathrm{s}}^{* *} \ell^{-} \bar{\nu} \mathrm{X}$. Decays of $\mathrm{D}_{\mathrm{s}}^{* *}$ to $\mathrm{DK}$ or $\mathrm{D}^{*} \mathrm{~K}$ have been observed [3], the decays to $\mathrm{D}_{\mathrm{s}} \pi$ being forbidden by isospin conservation. Assuming that $30 \pm 10 \%$ of semileptonic $B_{s}^{0}$ decays involve $D_{s}^{* *}$ production, consistent with the relative production rate of $D^{* *}$ mesons in semileptonic $B_{d}^{0}$ and $\mathrm{B}^{+}$decays [26], and taking $B\left(\overline{\mathrm{B}}_{\mathrm{s}}^{0} \rightarrow \ell^{-} \bar{\nu} \mathrm{X}\right)$ to be $11 \pm 2 \%$, we obtain $B\left(\mathrm{~b} \rightarrow \overline{\mathrm{B}}_{\mathrm{s}}^{0}\right)=0.17 \pm 0.06$.

Alternatively, information on $B\left(\mathrm{~b} \rightarrow \overline{\mathrm{B}}_{\mathrm{s}}^{0}\right)$ can be obtained from the time-integrated measurement, $\bar{\chi}=f_{\mathrm{s}} \chi_{\mathrm{s}}+f_{\mathrm{d}} \chi_{\mathrm{d}}$. Using $\bar{\chi}=0.119 \pm 0.009$ quoted in [27] and $\chi_{\mathrm{d}}$ from [2], and assuming that the rate of leptons produced from $\mathrm{B}_{\mathrm{d}}^{0}$ and $\mathrm{B}^{+}$decays are equal within $10 \%$, we obtain $B\left(\mathrm{~b} \rightarrow \overline{\mathrm{B}}_{\mathrm{s}}^{0}\right)=0.11 \pm 0.03$ if $\chi_{\mathrm{s}}$ is close to 0.5 . As 0.5 is the maximum possible value for $\chi_{\mathrm{s}}$, this constraint can be viewed as a lower limit on $B\left(\mathrm{~b} \rightarrow \overline{\mathrm{B}}_{\mathrm{s}}^{0}\right)$.

An average of these two values is $B\left(\mathrm{~b} \rightarrow \overline{\mathrm{B}}_{\mathrm{s}}^{0}\right)=0.13 \pm 0.03$, in good agreement with the range $0.12 \pm 0.036$ assumed in this analysis. The uncertainty assumed is larger than the error on this average, reflecting uncertainty on the assumptions input.

Acknowledgements:

It is a pleasure to thank the SL Division for the efficient operation of the LEP accelerator, the precise information on the absolute energy, and their continuing close cooperation with our experimental group. In addition to the support staff at our own institutions we are pleased to acknowledge the

Department of Energy, USA,

National Science Foundation, USA,

Particle Physics and Astronomy Research Council, UK,

Natural Sciences and Engineering Research Council, Canada,

Fussefeld Foundation,

Israel Ministry of Science,

Israel Science Foundation, administered by the Israel Academy of Science and Humanities, Minerva Gesellschaft, Japanese Ministry of Education, Science and Culture (the Monbusho) and a grant under the Monbusho International Science Research Program,

German Israeli Bi-national Science Foundation (GIF),

Direction des Sciences de la Matière du Commissariat à l'Energie Atomique, France,

Bundesministerium für Forschung und Technologie, Germany,

National Research Council of Canada,

A.P. Sloan Foundation and Junta Nacional de Investigação Científica e Tecnológica, Portugal. 


\section{References}

[1] See for example: P. Franzini, Physics Reports 173 (1989) 1.

[2] ARGUS Collaboration, H. Albrecht et al., Z. Phys. C 55 (1992) 357;

ARGUS Collaboration, H. Albrecht et al., Phys. Lett. B 324 (1994) 249;

CLEO Collaboration, J. Bartelt et al., Phys. Rev. Lett. 71 (1993) 1680.

[3] Particle Data Group, 'Review of Particle Properties', Phys. Rev. D 50 (1994) 1173.

[4] ALEPH Collaboration, D. Buskulic et al., Phys. Lett. B 313 (1993) 498;

DELPHI Collaboration, P. Abreu et al., Phys. Lett. B 338 (1994) 409.

[5] OPAL Collaboration, R. Akers et al., Phys. Lett. B 336 (1994) 585.

[6] OPAL Collaboration, R. Akers et al., Phys. Lett. B 327 (1994) 411.

[7] ALEPH Collaboration, D. Buskulic et al., Phys. Lett. B 322 (1994) 441.

[8] Y. Nir, Phys. Lett. B 327 (1994) 85;

A. Ali and D. London, CERN-TH/7398-94, submitted to Z. Phys. C.

[9] OPAL Collaboration, K. Ahmet et al., Nucl. Inst. and Meth. A 305 (1991) 275.

[10] P. Allport et al., Nucl. Inst. and Meth. A 324 (1993) 34;

P. Allport et al., Nucl. Inst. and Meth. A 346 (1994) 476.

[11] OPAL Collaboration, G. Alexander et al., Z. Phys. C 52 (1991) 175.

[12] OPAL Collaboration, R. Akers et al., Z. Phys. C 63 (1994) 197.

The jet finding parameters $\epsilon$ and $R$ were set to $5.0 \mathrm{GeV}$ and 0.65 , respectively.

[13] T. Sjöstrand, Comp. Phys. Comm. 39 (1986) 347;

M. Bengtsson and T. Sjöstrand, Comp. Phys. Comm. 43 (1987) 367;

M. Bengtsson and T. Sjöstrand, Nucl. Phys. B 289 (1987) 810;

T. Sjöstrand, CERN-TH/6488-92.

[14] Parameter values were tuned to describe global event shape variables:

OPAL Collaboration, M. Akrawy et al., Z. Phys. C 47 (1990) 505.

[15] J. Allison et al., Nucl. Instrum. Methods A 317 (1992) 47.

[16] C. Peterson, D. Schlatter, I. Schmitt and P. Zerwas, Phys. Rev. D 27 (1983) 105.

[17] D. Bardin et al., ZFITTER, An Analytical Program for Fermion Pair Production in $\mathrm{e}^{+} \mathrm{e}^{-}$Annihilation, CERN-TH/6443-92.

For this prediction, the $\mathrm{Z}^{0}$, top quark, and Higgs boson masses are set to $M_{\mathrm{Z}^{0}}=91.18 \mathrm{GeV}, M_{\text {top }}=150 \mathrm{GeV}$ and $M_{\text {Higgs }}=300 \mathrm{GeV}$, and $\alpha_{s}=0.12$.

[18] OPAL Collaboration, P. Acton et al., Z. Phys. C 58 (1993) 523.

[19] OPAL Collaboration, R. Akers et al., Z. Phys. C 60 (1993) 199. 
[20] ALEPH Collaboration, D. Buskulic et al., Phys. Lett. B 322 (1994) 275;

ALEPH Collaboration, D. Buskulic et al., Phys. Lett. B 314 (1993) 459;

ALEPH Collaboration, D. Buskulic et al., Phys. Lett. B 307 (1993) 194;

ALEPH Collaboration, D. Buskulic et al., Phys. Lett. B 295 (1992) 396;

DELPHI Collaboration, P. Abreu et al., Z. Phys. C 63 (1994) 3;

DELPHI Collaboration, P. Abreu et al., Phys. Lett. B 312 (1993) 253;

DELPHI Collaboration, P. Abreu et al., Phys. Lett. B 311 (1993) 379;

OPAL Collaboration, P. Acton et al., Z. Phys. C 60 (1993) 217;

OPAL Collaboration, R. Akers et al., Phys. Lett. B 316 (1993) 435;

OPAL Collaboration, P. Acton et al., Phys. Lett. B 312 (1993) 501;

OPAL Collaboration, P. Acton et al., Phys. Lett. B 307 (1993) 247.

[21] CDF Collaboration, F. Abe et al., Phys. Rev. Lett. 71 (1993) 3421;

CDF Collaboration, F. Abe et al., Phys. Rev. Lett. 72 (1994) 3456.

The following paper appeared during the final editing of this paper, and the results were not included:

CDF Collaboration, F. Abe et al., FERMILAB-PUB-94-420-E, January 1995.

[22] The production of strange quark pairs in the fragmentation was tuned to reproduce the inclusive $\mathrm{K}_{\mathrm{S}}^{0}$ yield measured in

OPAL Collaboration, G. Alexander et al., Phys. Lett. B 264 (1991) 467.

[23] OPAL Collaboration, P. Acton et al., Phys. Lett. B 273 (1991) 355.

[24] OPAL Collaboration, R. Akers et al., Z. Phys. C 65 (1995) 17.

[25] ALEPH Collaboration, D. Buskulic et al., Phys. Lett. B 294 (1992) 145;

OPAL Collaboration, P. Acton et al., Phys. Lett. B 295 (1992) 357.

[26] OPAL Collaboration, R. Akers et al., CERN-PPE/95-02, submitted to Z. Phys. C;

ALEPH Collaboration, D. Buskulic et al., CERN-PPE/94-173, submitted to Phys. Lett. B.

[27] R. Forty, CERN-PPE/94-154, to appear in Proc. of ICHEP Conference, Glasgow, July 1994. 


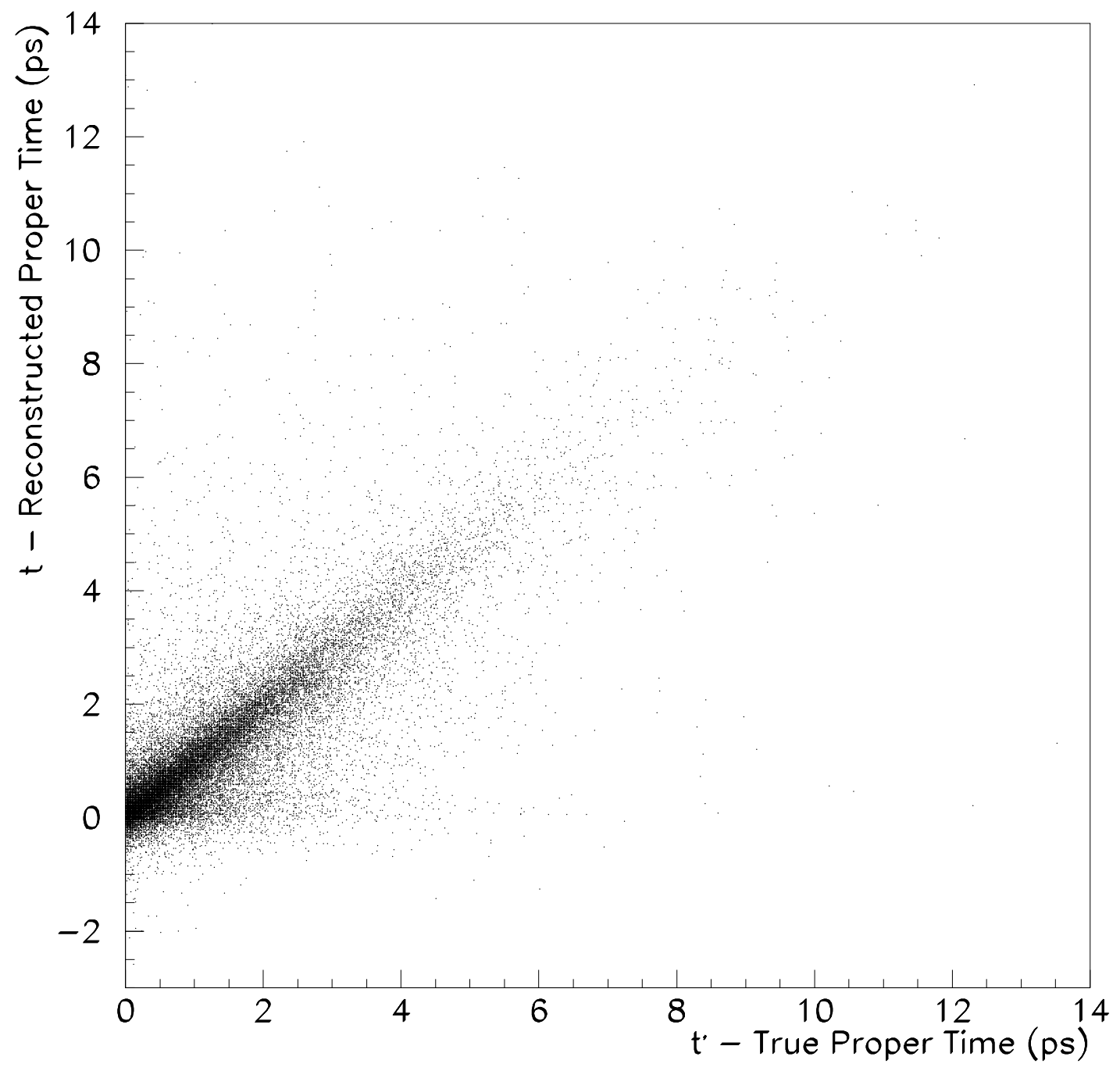

Figure 1: The reconstructed proper time is plotted versus the true proper time for leptons from primary $\mathrm{b}$ hadron decays in the Monte Carlo. 

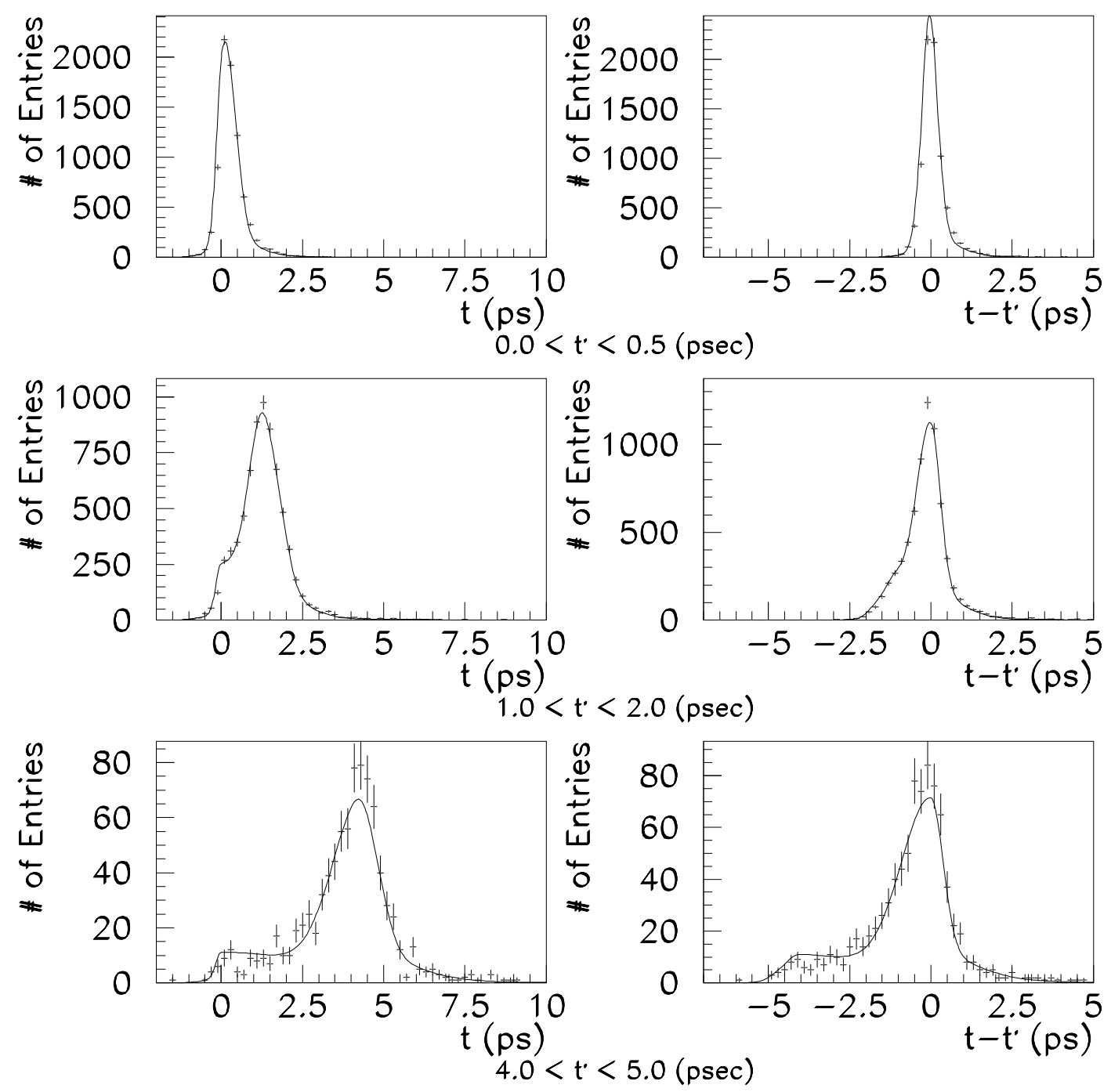

Figure 2: The distributions of reconstructed proper time, $t$, and $t-t^{\prime}$ in three slices of the true proper time $t^{\prime}$ for leptons from primary b hadron decays in the Monte Carlo. Also shown is the parametrisation of these distributions, described in Section 5. 


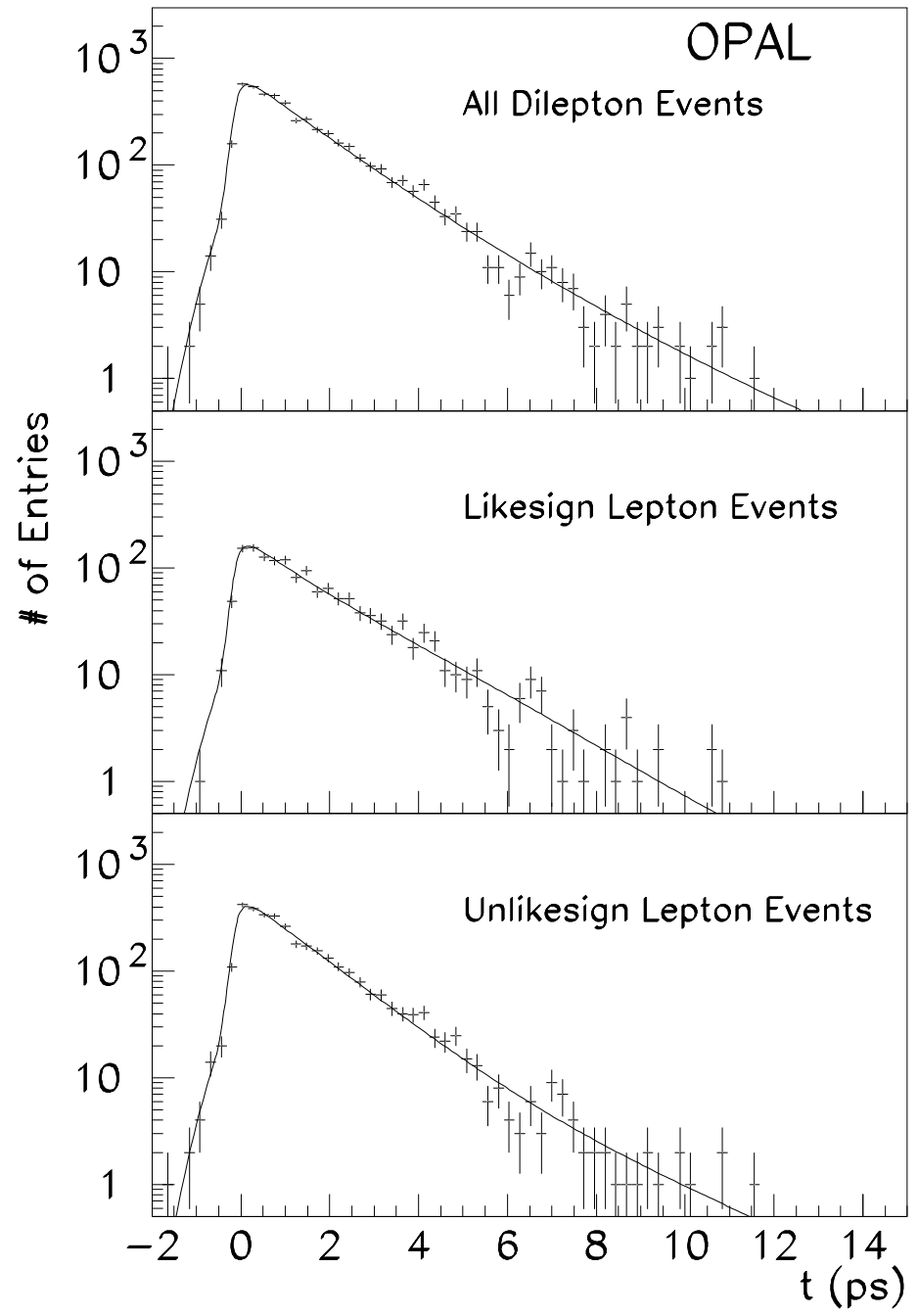

Figure 3: The proper time distributions for all leptons in dilepton events (top) for which a vertex was found, and for those leptons in like-sign (centre) and unlike-sign (bottom) events. The curves represent the results of the maximum likelihood fit. 


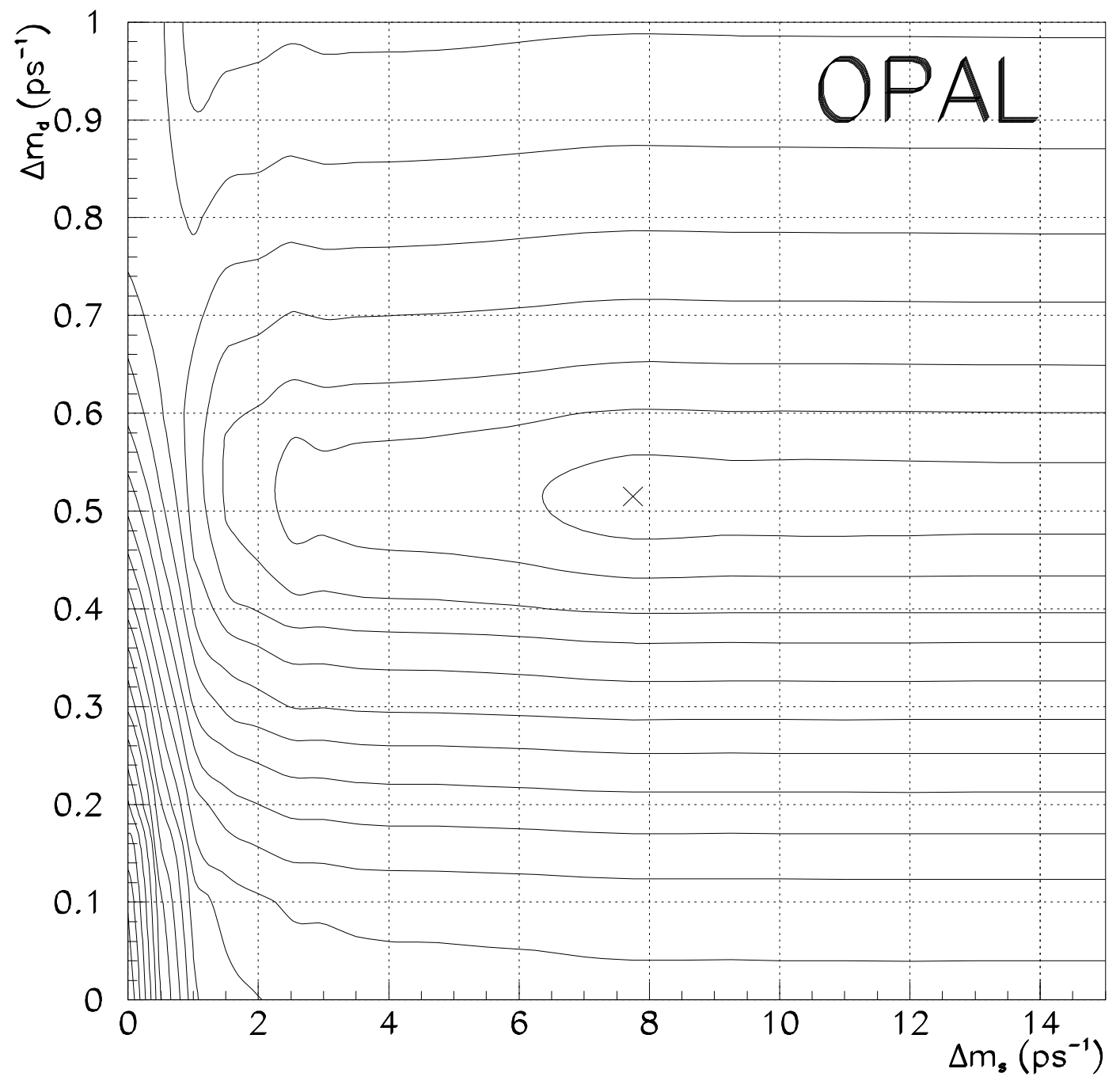

Figure 4: The log-likelihood contours in the $\Delta m_{\mathrm{d}}-\Delta m_{\mathrm{s}}$ plane for the OPAL data. The contour lines are at $\ln \left(\mathcal{L}_{\max } / \mathcal{L}\right)=0.5,2,4.5 \ldots$, corresponding to $1 \sigma, 2 \sigma, 3 \sigma$, etc. 


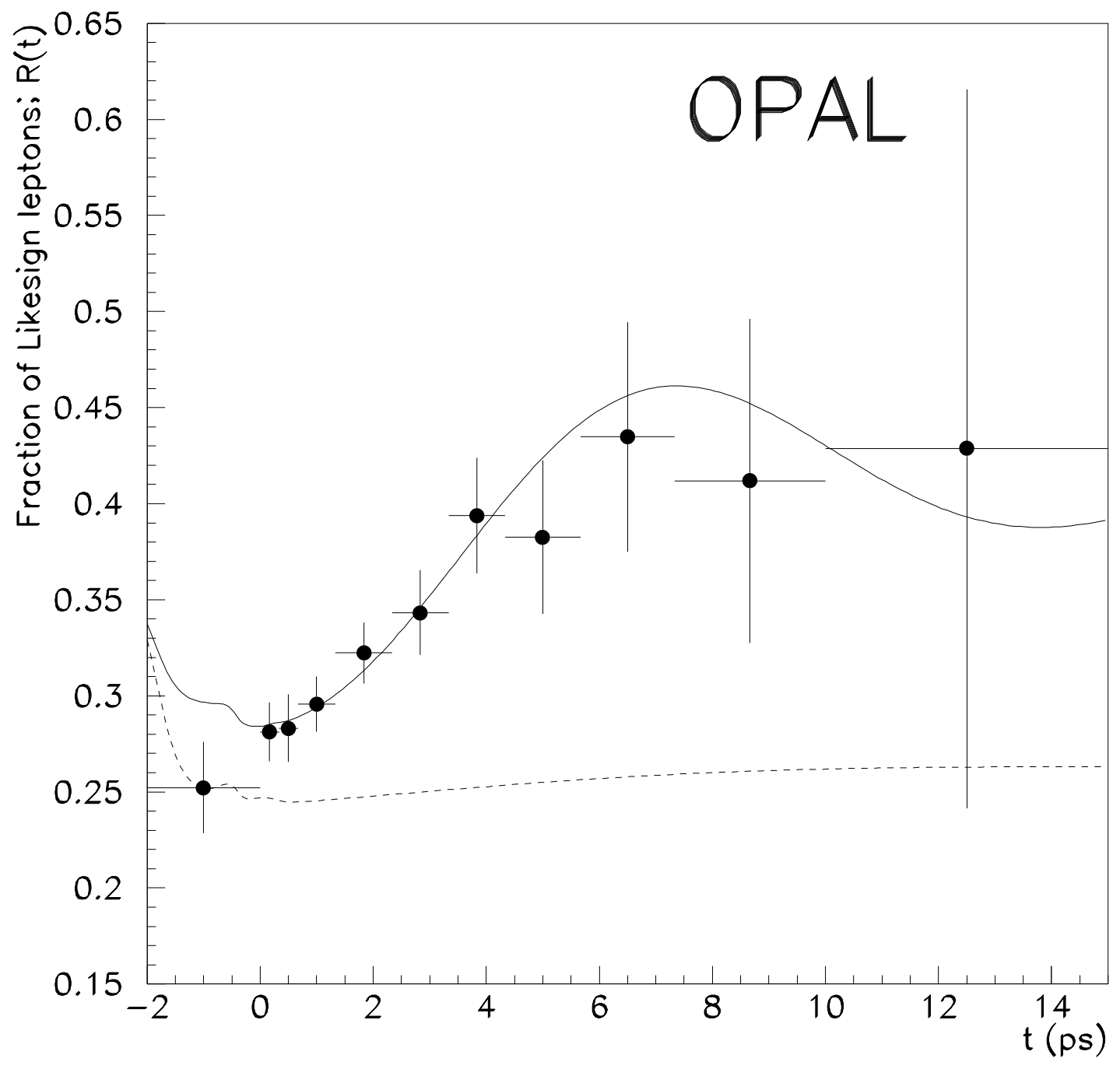

Figure 5: The fraction of like-sign leptons as a function of proper decay time: $\mathcal{R}(t)$. The solid curve represents the expectation with $\Delta m_{\mathrm{d}}$ set to $0.462 \mathrm{ps}^{-1}$ and $\Delta m_{\mathrm{s}}$ set to $10.0 \mathrm{ps}^{-1}$. The dashed curve represents the prediction with no $\mathrm{B}_{\mathrm{d}}^{0}$ mixing. 


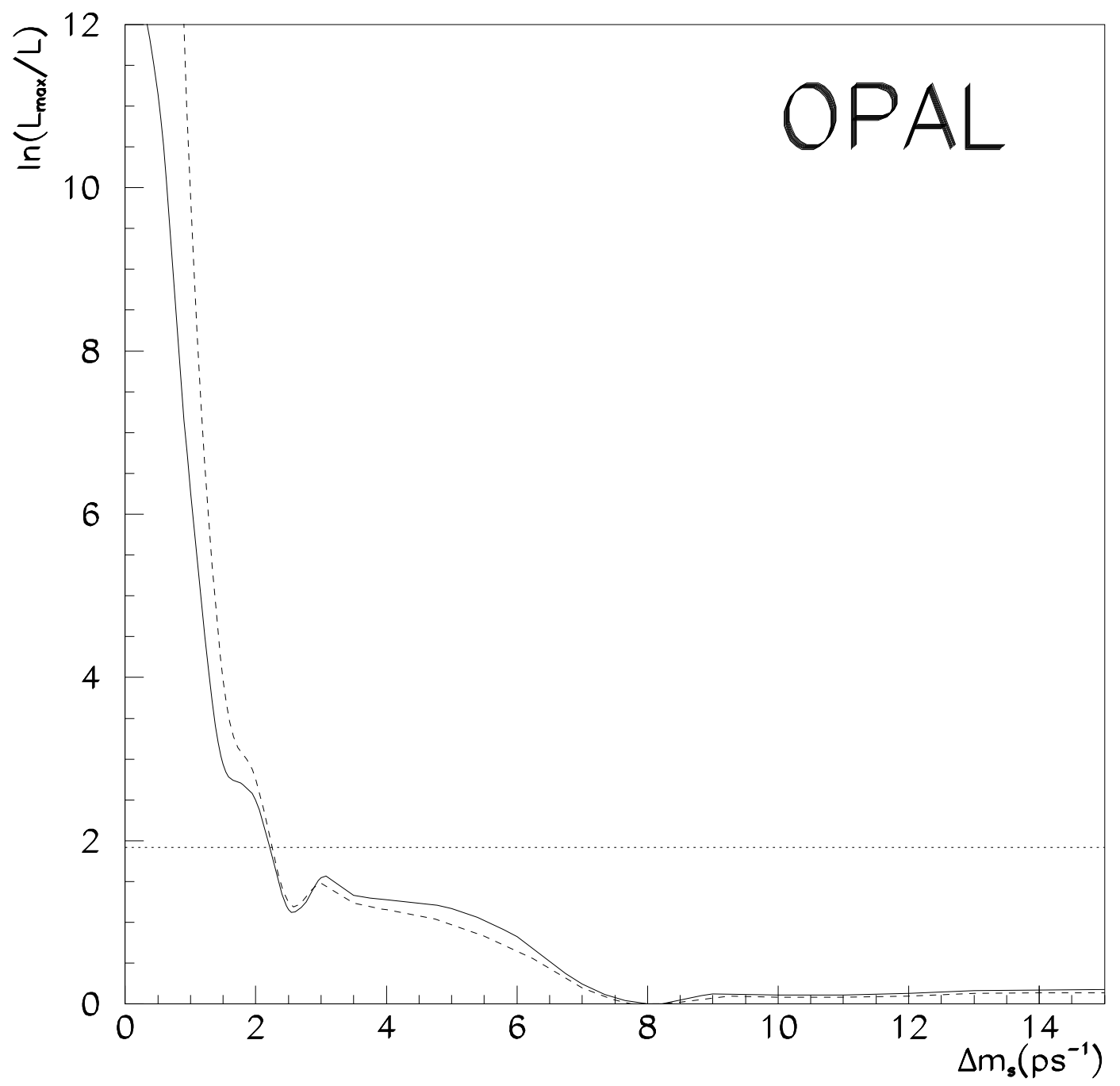

Figure 6: The difference in log-likelihood from the maximum value is shown as a function of $\Delta m_{s}$. The solid curve includes the effect of systematic errors, while the dashed curve is purely based on statistics. The scan was performed at a discrete set of points, and the curves merely connect adjacent scan points. 

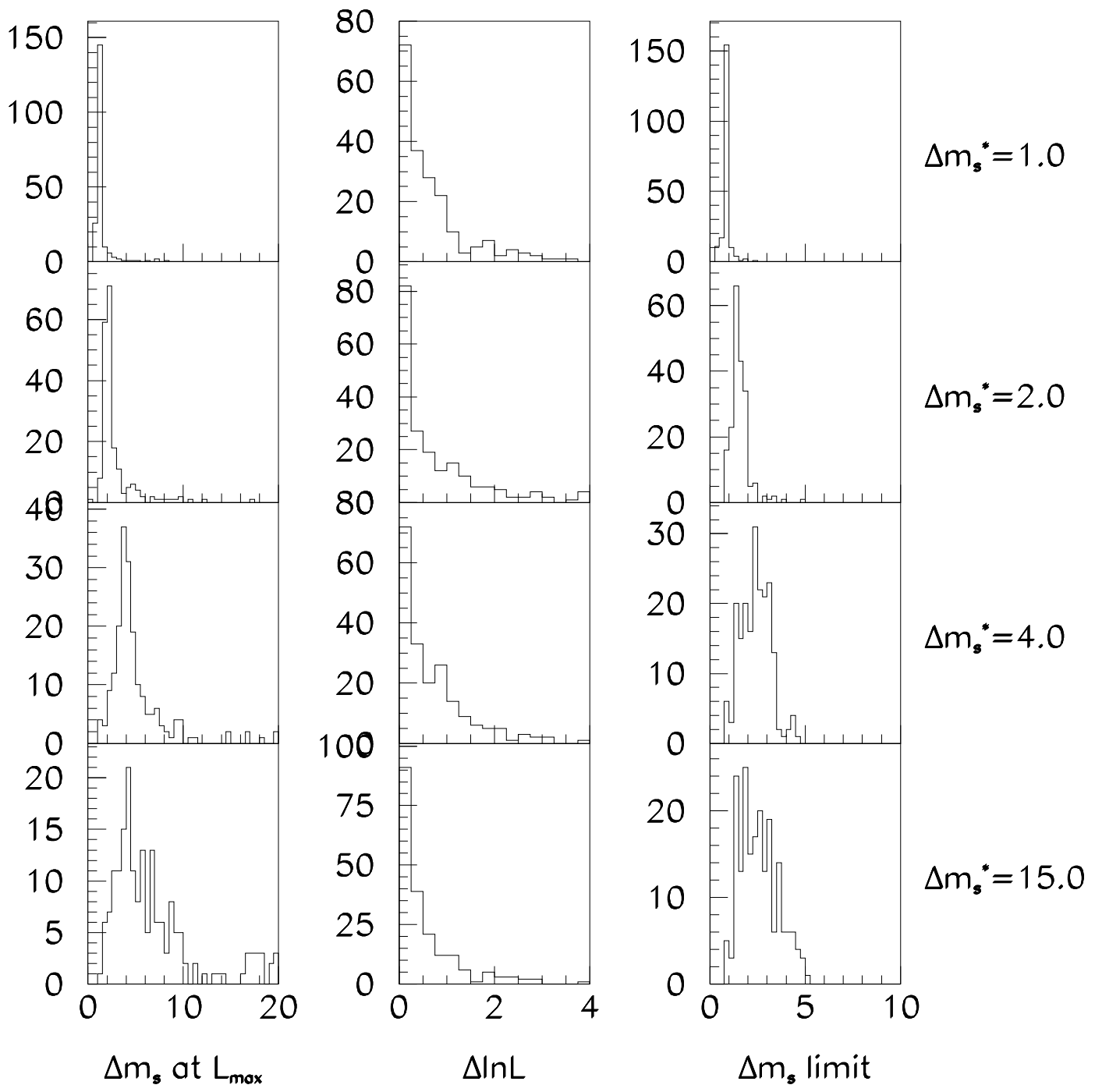

Figure 7: The results of fits to 200 toy Monte Carlo datasets are shown. The $\Delta m_{\mathrm{s}}^{*}$ value indicates the generated value of $\Delta m_{\mathrm{s}}$ in $\mathrm{ps}^{-1}$. The three quantities plotted for each $\Delta m_{\mathrm{s}}^{*}$ value are the fitted value of $\Delta m_{\mathrm{s}}$, the difference between the log-likelihood values at the fitted maximum and at the generated value, and the statistical lower limit one would derive for $\Delta m_{\mathrm{s}}$ on each trial. 